\title{
Effective flow surface of porous materials with two populations of voids under internal pressure: II. full-field simulations
}

\author{
Pierre-Guy Vincent ${ }^{\mathrm{a}, \mathrm{b}}$, Pierre Suquete, ${ }^{\mathrm{c}, *}$, Yann Monerie ${ }^{\mathrm{a}, \mathrm{b}, 1}$, Hervé Moulinec $^{\mathrm{c}}$ \\ ${ }^{a}$ Institut de Radioprotection et de Sûreté Nucléaire, B.P. 3, 13115 Saint-Paul-lez-Durance Cedex, France \\ ${ }^{b}$ Laboratoire de Micromécanique et d'Intégrité des Structures, IRSN-CNRS-UMII, B.P. 3, 13115 \\ Saint-Paul-lez-Durance Cedex, France \\ ${ }^{c}$ Laboratoire de Mécanique et d'Acoustique, CNRS, UPR 7051, Aix-Marseille Univ, Centrale Marseille, 31, \\ Chemin Joseph Aiguier, 13402 Marseille Cedex 20, France
}

\begin{abstract}
This study is devoted to the effective plastic flow surface of a bi-porous material saturated by a fluid. Highly irradiated uranium dioxide is a typical example of such a material. In part I of this study, a GTN-type approximation of the effective plastic flow surface has been derived. In this second part, the predictions of this new model are compared with full-field numerical simulations performed with a numerical method based on Fast Fourier Transforms. This method is successfully applied to voided materials with a Gurson matrix where the voids are subjected to internal pressure. Different microstructures containing a large number of spherical or ellipsoidal voids are investigated. The deviation from isotropy of their mechanical response is measured by a new criterion.
\end{abstract}

Keywords: A. Ductility, B. Microstructures, B. Ideally plastic material, B. Porous material, C. Numerical algorithms

\section{Introduction}

In the second part of this study devoted to the mechanical behavior of highly irradiated uranium dioxide fuel $\left(\mathrm{UO}_{2}\right)$ under accident conditions, the predictions of the analytical models proposed in the first part are compared to full-field simulations. As recalled in part I of this paper, $\mathrm{UO}_{2}$ exhibits a very specific microstructure with two populations of voids of rather different sizes and shapes: a first population of voids, almost spherical in shape, is observed inside the grains at the lowest scale (intragranular voids), and a second population of voids, almost lenticular and located at the grain boundaries can be found at a larger scale (intergranular voids). During a reactivity initiated accident (RIA), the temperature of the

\footnotetext{
*Corresponding author. Tel: +334911642 08; fax: +33491164481

Email addresses: pierre-guy.vincent@irsn.fr (Pierre-Guy Vincent), suquet@lma.cnrs-mrs.fr (Pierre Suquet), yann.monerie@irsn.fr (Yann Monerie), moulinec@lma.cnrs-mrs.fr (Hervé Moulinec)

${ }^{1}$ Present address: Laboratoire de Mécanique et Génie Civil, Université Montpellier 2, CNRS, CC 048 Place Eugène Bataillon, 34095 Montpellier Cedex, France
}

Preprint submitted to International Journal of Plasticity

December 2, 2013 
fuel increases abruptly, inducing a thermal dilatation of the material and a strong increase of the pressure in the voids due to the presence of fission gases confined inside these bubbles. At the mesoscale, the matrix (where intragranular voids are already smeared out) is modelled as a pressure-sensitive ductile material governed by the Gurson-Tvergaard-Needleman (GTN) criterion (Gurson, 1977; Koplik and Needleman, 1988; Tvergaard, 1990). The objective of this study is to derive a criterion describing the effective flow surface of the voided material at the macroscopic scale when the intergranular voids are ellipsoidal and subjected to an internal pressure.

The analytical Gurson-like criterion (or GTN criterion) proposed in the first part of this study (Vincent et al., 2013) takes the form :

$$
\frac{1}{\beta}\left(\frac{\Sigma_{e q}}{\sigma_{0}}\right)^{2}+\frac{1}{\alpha} \cosh \left(\frac{3}{2}\left(\frac{\Sigma_{m}}{\sigma_{0}}-\gamma\right)\right)-1=0,
$$

where $\alpha, \beta$, and $\gamma$ are three functions of the parameters of the problem, namely $f_{b}$ the void volume fraction of the spherical intragranular voids of very small size, $f_{e}$ and $w$ the void volume fraction and aspect-ratio of the ellipsoidal (oblate) intergranular voids, $p_{b}$ and $p_{e}$ the internal pressures in the intragranular and intergranular voids, respectively (see part I of this study, Vincent et al., 2013, for more details). Fritzen et al. (2013) have recently published a similar study for voided materials with a pressure-sensitive matrix of Green type. The present study differs from the latter one in three aspects: first the matrix in the present study is a Gurson material, second the voids are randomly oriented ellipsoids and third they are subjected to an internal pressure.

The approach followed to derive (1) is based on a study of an elementary volume element made of a single hollow ellipsoid and extended to an assemblage of self-similar randomly oriented ellipsoidal voids, as schematized in Figure 1.
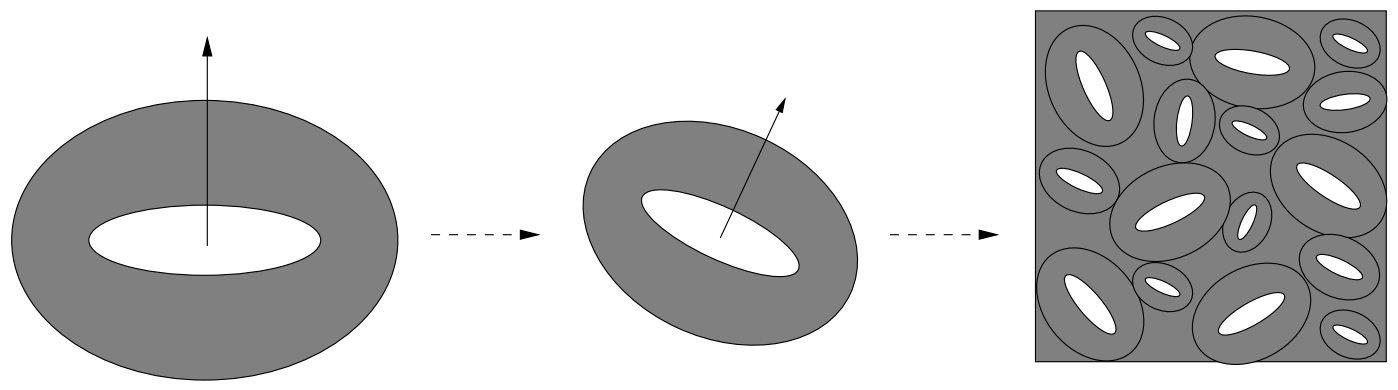

Figure 1: Assemblage of self-similar randomly oriented hollow ellipsoids (from (Vincent et al., 2009)). Unit pattern (left). Rotated and dilated hollow ellipsoid (center). Representative volume element (right).

Since, to the best of the author's knowledge, no comparison with experimental data is available to assess the validity of the analytical model (1), its predictions are compared here with three-dimensional full-field simulations, performed with a numerical method based on 
Fast Fourier Transforms. This iterative method was proposed by Moulinec and Suquet $(1994,1998)$ for microstructures subjected to periodic boundary conditions and does not require, at least explicitly, any meshing of the microstructure.

The organization of the paper is as follows:

- The procedure followed to generate different microstructures used in the full-field simulations is presented in section 2.1. These microstructures must contain a large number of voids to approach overall isotropy as closely as possible. An original (to the best of our knowledge) criterion for measuring deviation from isotropy is introduced.

- A reminder on the FFT method, and its variant based on augmented Lagrangians is given in section 3 .

- Finally the results of the full-field simulations for spherical and ellipsoidal voids are presented in sections 4.2 and 4.3 and compared with the analytical criterion (1). The agreement is found to be good, not only for the effective flow surface but also for the average dilatation-rate in the matrix which governs the evolution of the void volume fraction of both population of voids.

\section{Microstructures}

\subsection{Microstructure generation}

The full-field simulations are performed on artificially generated microstructures. The unit-cell is a unit cube. The microstructures are generated in two steps.

1. First the unit-cell is filled with spheres using an algorithm inspired by Molecular Dynamics. This algorithm proceeds iteratively. First, the spheres are randomly placed in the volume and can freely overlap. Then the distance of interpenetration between each sphere and its neighbors is calculated as the sum of the radius of the sphere and of its neighbors minus the distance between the centers of the two spheres (or zero if the spheres do not interpenetrate). For each sphere all the interprenetration distances are summed up and the center of the sphere is moved by a given fraction of this sum augmented by a small random displacement. The iterative process is stopped as soon as no interpenetration is detected between spheres. The volume fraction which can be reached by the classical Random Sequential Addition algorithm (Torquato, 2003) does not exceed $40 \%$ whereas the filling rate obtained with the above MD algorithm is about $60 \%$ for monodisperse spheres and higher for polydisperse spheres.

2. In a second step, a unit void, either a spherical void or an ellipsoidal void with random orientation, is located at the center of each sphere. All single hollow elements are selfsimilar and the void volume fraction in each of them is identical, chosen in such a way that the overall void volume fraction $f_{e}$ is reached. Therefore each void is surrounded by a shell of matrix with a width proportional to the void size. 
The outer spheres may have the same diameter (monodisperse spheres) and then all the voids have the same size (but their orientation is different when they are not spherical). Or they may have different diameters (polydisperse spheres) in which case the voids have different sizes. The close-packing volume fraction of the spheres is larger in the polydisperse case than in the monodisperse case and can approach $f_{e}=1$, at least theoretically, if an infinite number of sizes can be used. Obviously in practice the number of different sizes for the spheres is limited.

For spherical voids, where no problem of orientation of the voids arises, polydisperse spheres (and voids) were chosen with 3 different sizes of voids. Two typical microstructures are shown in Figure 2. The realization on the left contains 73 small voids, 12 medium size voids, and 8 large voids. The realization on the right contains 9,3 and 1 voids in each class of size (the size ratios with respect to the smallest size are respectively 1.0, 1.8, and 3.3).
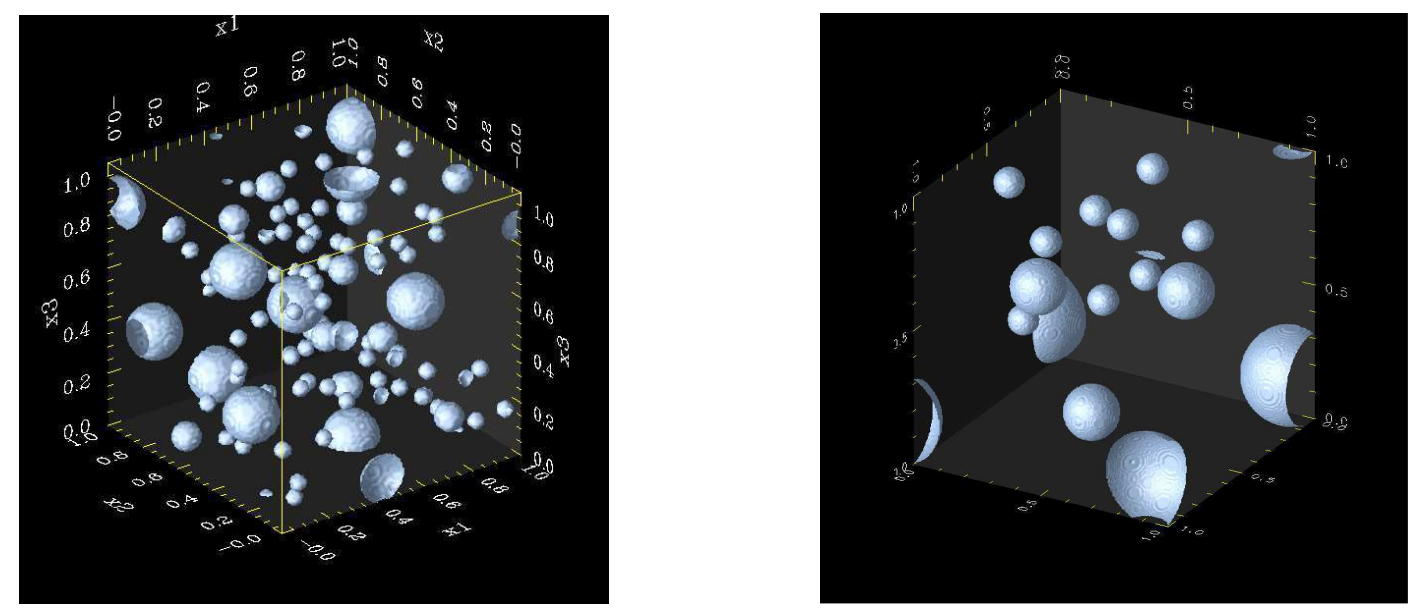

Figure 2: Periodic unit cells used for the FFT simulations of a microstructure with spherical in shape voids. Total void volume fraction $f_{e}=0.04$.

The monodisperse situation is the choice that was made for ellipsoidal voids to ensure that all orientations are equally represented in the microstructure with the same weight (at least to a good accuracy). Three different realizations are shown in Figure 3 with a void aspect ratio $w=1 / 5$. Attention is restricted to oblate spheroids where the third axis $a_{1}$ is smaller than the two equal axes $b_{1}$ (the aspect ratio $w$ is defined as $a_{1} / b_{1}$ ). Each of these realizations contains 511 voids.

\subsection{Spatial resolution}

The FFT method does not require a specific meshing of the unit cell but still makes use of a sampling of the image describing the microstructure. The microstructure is given as a digital image, provided either by an experimental device (computed tomography, 3D X-ray diffraction or any other mean), or generated artificially (see Lebensohn et al., 2011, for a review of possible applications of the method). The image consists of equally sized voxels 


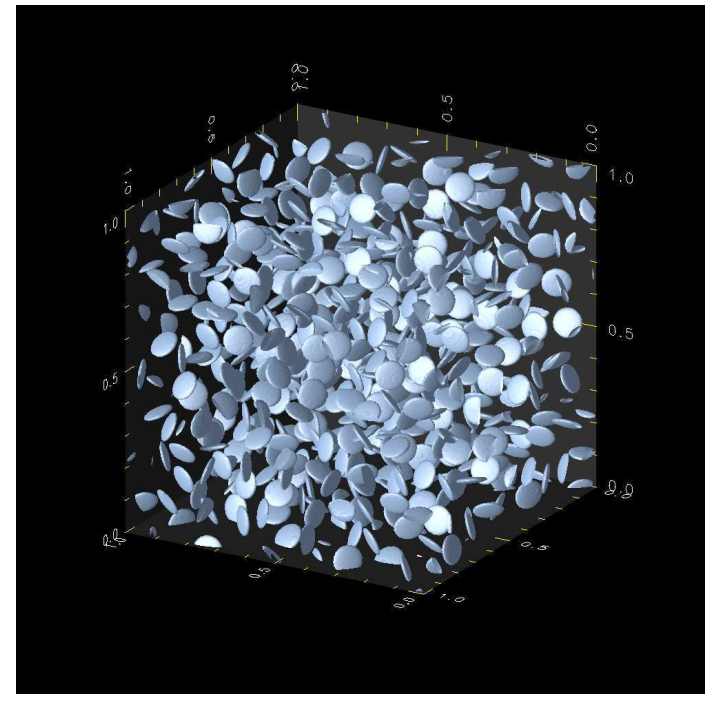

Microstructure \# 1

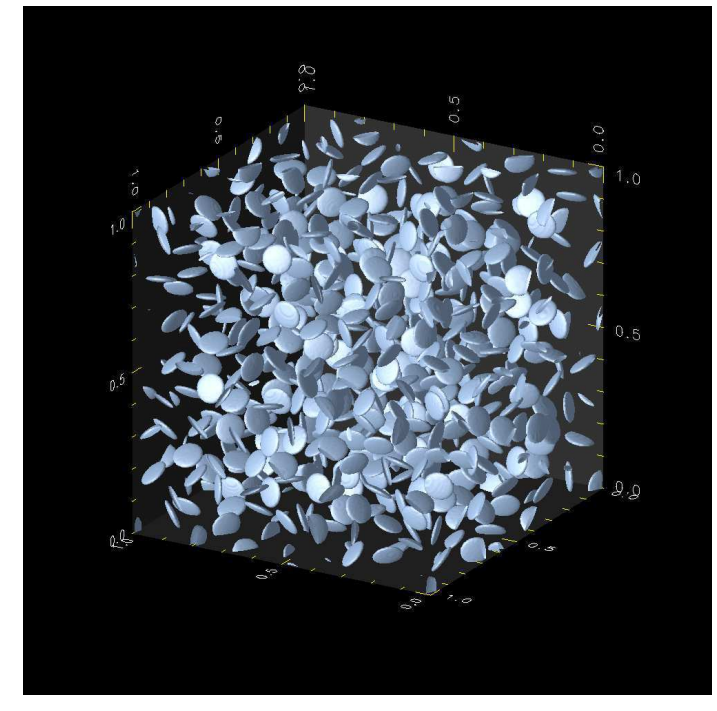

Microstructure \# 2

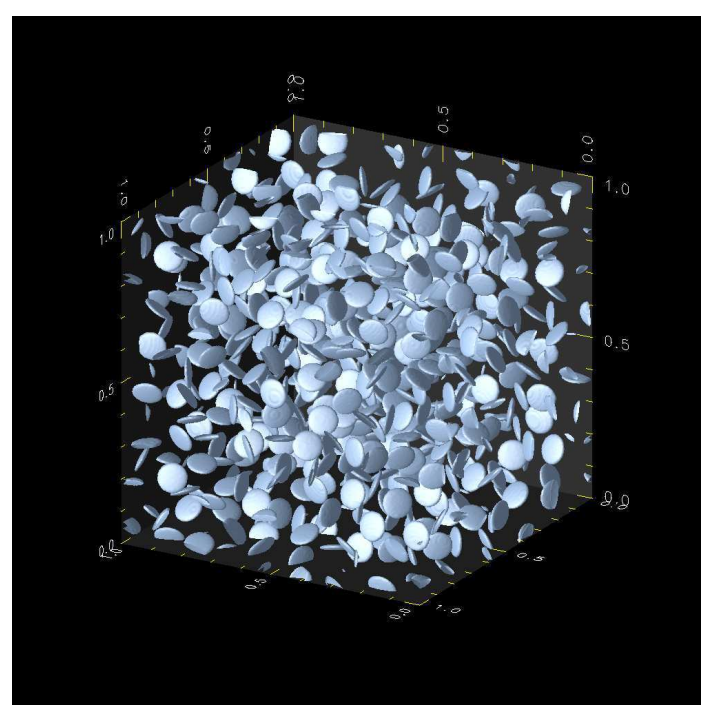

Microstructure \# 3

Figure 3: Spheroidal intergranular voids. Three different realizations of the unit-cell containing 511 ellipsoidal voids with total volume fraction $f_{e}=0.04$. All ellipsoids have the same size. Aspect ratio of the ellipsoids: $w=1 / 5$. 
and its resolution is measured by the number of voxels, so that even if no specific meshing is formally required, there is an inherent spatial resolution of the image related to the number of voxels in the image. The sampling is regular, allowing for the use of commonly available FFT packages, and the unit-cell is discretized into $N \times N \times N$ voxels of identical size. $N$ is limited by the memory capability of the computer on which the simulations are performed. The largest images used in the present simulations are discretized into $512^{3}$ voxels (the corresponding unit-cells are shown in Figure 3). The question of spatial resolution is then closely related to determining the number of voids which can be distributed in the unit-cell with a given sampling. This question pertains to determining how many voxels should be used for an elementary pattern such as the one shown in Figure 4 (right) to capture accurately the field fluctuations (stress and strain) in the matrix around each void. This is a technical issue which should be addressed, ideally, realization by realization, by conducting parametric studies in which the number of voxels is increased until stationarity of the quantities of interest (effective properties, local fields) is reached. Following this procedure would require a formidable computational effort. Instead of that what was done here was to examine a cubic unit-cell containing a single spherical void at its center and we determined how many voxels are required to achieve a reasonable compromise for a single void. Detailed results are reported in Appendix B. The cubic unit-cell was discretized into $32^{3}, 64^{3}, 128^{3}$ voxels and convergence of the effective flow surface with the spatial resolution was examined. It was found that the intermediate spatial discretization $64^{3}$ voxels was sufficient to ensure results which deviate from less than $1 \%$ compared to the results at the highest resolution. Therefore it was decided to distribute $M$ voids in the unit-cell, with $M$ lower than $(N / 64)^{3}$, so that each void and its surrounding shell of matrix could be discretized into more than $64^{3}$ voxels (in average, depending on the void size) and the same criterion was applied for ellipsoidal voids. For instance when $N=512$, the number of voids in the unit-cell should be less than 512 (hence the 511 ellipsoidal voids in Figure 3).

\subsection{Representativeness}

The question of representativeness of the results of the full-field simulations is a difficult issue: how many realizations should be investigated and how big should they be? No attempt has been made in the present study to fully, and definitively, answer these questions. First because answering them would require a mathematical background which, to the best of the authors' knowledge, does not exist for nonlinear materials. Second because the computational effort necessary to explore systematically the effect of the size of the representative volume element, for all loading cases and all configurations, would be prohibitive. The results presented here, even if restricted to a limited number of realizations, correspond to 334 different full-field calculations. The CPU time for one full-field simulation with the largest realizations was of the order of 35 hours on 2 Intel ${ }^{\circledR}$ Xeon ${ }^{\circledR}$ processors E7-8870 2.4 GHz (20 cores) with 70 GB RAM.

Therefore, in the absence of a rational guideline to decide how many realizations are necessary to achieve representativity, the results and comparisons reported in the sequel are 

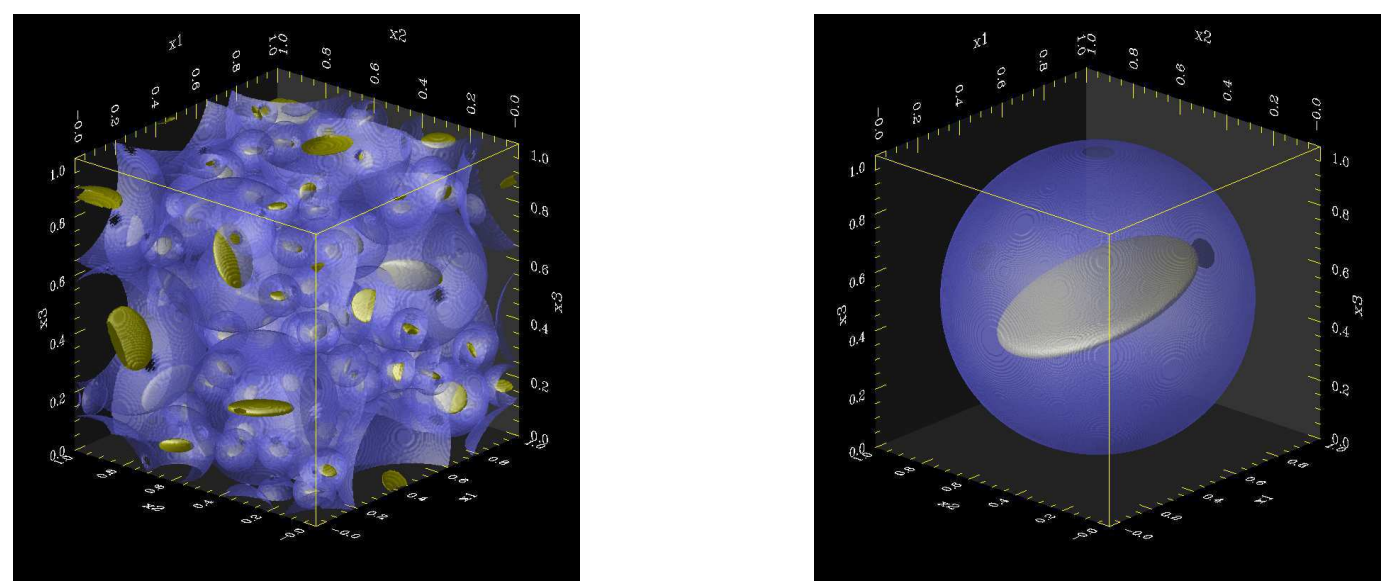

Figure 4: Left: unit cube filled with spheres (in gold) containing a central ellipsoidal void (purple). Right: Elementary pattern

largely based on the authors' experience to decide whether the results are representative. Admittedly, this way of proceeding based on experience can be disputed. But it can be motivated by a few general comments drawing the attention of the reader to the fact that the above question of representativeness is not well-posed as such and should be formulated in a more precise way to obtain a more specific answer.

First the notion of representativeness is an asymptotic notion, in the sense that exact stationarity of the quantities of interest can only be reached in the limit of an infinite domain. For finite volumes, such as the ones corresponding to specific realizations of a unitcell, one can only require that the deviation from stationarity should be less than a given threshold (a few percents usually). So a better formulation of the question should be: how big should a realization be, or how many realizations of a prescribed size are required to attain stationarity of a quantity of interest with an error less than a given threshold? Even this question is difficult as the exact stationary value is not known, and therefore the error must be inferred from the simulations themselves. We shall then report the discrepancy between different simulations performed on different realizations (and not only the average results).

Secondly the answer depends on the "quantity of interest". In the present context, stationarity is understood for the effective flow surface (up to a prescribed error). This does not necessarily ensure stationarity for other quantities such as the void growth-rate, the strain fluctuations in the matrix, or the maximum strain attained locally in the matrix 
(among other examples of quantities of interest). Obviously the more local the information for which stationarity is seeked, the larger the volume element should be. Here we will discuss only the effective flow surfaces and estimate the distance between the flow surfaces obtained for different realizations by measuring the difference along radial paths in stress space. As a general comment, we point out that the notion of representativity is intimately related to a quantity of interest.

Obviously the size of the representative volume element also depends on the constitutive relations of the constituents. Our experience is that nonlinear materials require larger volume elements than linear materials (but this experience is not always shared by other researchers in the field and this question is left as an open question).

The conclusion is that the question of representativity cannot be answered thoroughly, either theoretically or computationally. A related question (not strictly equivalent) pertaining to macroscopic isotropy is easier to answer. It was indeed observed in the course of our simulations that when averaging the results over a given set of realizations, stationarity and isotropy where closely related. Since the voided materials investigated in this study are made of an isotropic matrix containing randomly oriented voids, macroscopic isotropy is a requirement for which the results should be checked.

\subsection{Deviation from isotropy}

There are several possible definitions of isotropy for a volume element. A first possible definition, which can be called geometrical isotropy, pertains to the geometrical arrangement of the phases and is assessed by inspecting the geometry of the volume element. This can be done by considering the covariograms of the phases, as proposed for instance in Kanit et al. (2003). A second possible definition of isotropy, which can be called mechanical isotropy, consists in assessing the isotropy of the mechanical response of the volume element under the application of mechanical loads, for given constitutive relations of the phases. The first definition is broader than the second one, in the sense that geometrical isotropy implies mechanical isotropy. However, as explained in more details in section 2.3, isotropy for nonlinear materials is rarely met exactly (by constrast with the linear case where a finite group of symmetry is sufficient to ensure isotropy) and therefore in both cases, geometrical or mechanical isotropy, a measure of deviation from isotropy has to be introduced. In the geometrical approach, based on the inspection of $2 \mathrm{~d}$-covariograms of specific cuttings of a $3 \mathrm{~d}$ image, the deviation from isotropy is difficult to define and remains mostly qualitative (a short study based on covariograms is reported in Appendix D). This is more easily achieved in the mechanical approach, where a quantitative measure of deviation from isotropy under different loadings can be introduced. This mechanical approach will be followed in the sequel.

Macroscopic isotropy imposes necessary conditions on the relations between the overall stress and the overall strain-rate obtained through full-field simulations. Consider a macroscopically isotropic, ideally plastic material. Its yielding properties can be described by 
means of a yield function $\mathcal{F}$ depending on the three invariants of $\boldsymbol{\Sigma}$ (the argument assumes that the yield criterion is smooth):

$$
\mathcal{F}(\Sigma)=\mathcal{F}\left(\Sigma_{m}, \Sigma_{e q}, J_{3}\right), \quad J_{3}=\frac{1}{3} \Sigma_{i j}^{d} \Sigma_{j k}^{d} \Sigma_{k i}^{d} .
$$

Assuming that $\mathcal{F}$ is differentiable, the overall strain-rate associated with this yield function by the normality rule reads as:

$$
\dot{\boldsymbol{E}}=\dot{\lambda} \frac{\partial \mathcal{F}}{\partial \Sigma_{m}}\left(\Sigma_{m}, \Sigma_{e q}, J_{3}\right) \frac{\partial \Sigma_{m}}{\partial \boldsymbol{\Sigma}}+\dot{\lambda} \frac{\partial \mathcal{F}}{\partial \Sigma_{e q}}\left(\Sigma_{m}, \Sigma_{e q}, J_{3}\right) \frac{\partial \Sigma_{e q}}{\partial \boldsymbol{\Sigma}}+\dot{\lambda} \frac{\partial \mathcal{F}}{\partial J_{3}}\left(\Sigma_{m}, \Sigma_{e q}, J_{3}\right) \frac{\partial J_{3}}{\partial \boldsymbol{\Sigma}}
$$

with

$$
\frac{\partial \Sigma_{m}}{\partial \boldsymbol{\Sigma}}=\frac{1}{3} \boldsymbol{i}, \quad \frac{\partial \Sigma_{e q}}{\partial \boldsymbol{\Sigma}}=\frac{3}{2} \frac{\boldsymbol{\Sigma}^{d}}{\Sigma_{e q}}, \quad \frac{\partial J_{3}}{\partial \boldsymbol{\Sigma}}=\boldsymbol{\Sigma}^{d} \cdot \boldsymbol{\Sigma}^{d}-\frac{2}{9} \Sigma_{e q}^{2} \boldsymbol{i} .
$$

Therefore the overall strain-rate can be split over three mutually orthogonal tensors $\left.\boldsymbol{V}_{i}\right|_{i=1,2,3}$ :

$$
\dot{\boldsymbol{E}}=\sum_{i=1}^{3} \dot{\xi}^{(i)} \boldsymbol{V}_{i}, \quad \boldsymbol{V}_{1}=\boldsymbol{i}, \quad \boldsymbol{V}_{2}=\boldsymbol{\Sigma}^{d}, \quad \boldsymbol{V}_{3}=\boldsymbol{\Sigma}^{d} \cdot \boldsymbol{\Sigma}^{d}-\frac{2}{9} \Sigma_{e q}^{2} \boldsymbol{i}-\frac{9}{2} \frac{J_{3}}{\Sigma_{e q}^{2}} \boldsymbol{\Sigma}^{d}
$$

where the $\dot{\xi}^{(i)}$ 's are scalars. The computed overall strain-rate $\dot{\boldsymbol{E}}^{\text {comp }}$ associated with a given overall stress state $\boldsymbol{\Sigma}$ located on the overall yield surface should conform to the decomposition (5). To check that this requirement is satisfied $\dot{\boldsymbol{E}}^{\text {comp }}$ is decomposed into a component parallel to the space spanned by the $\boldsymbol{V}_{i}$ 's and a component orthogonal to this space:

$$
\dot{\boldsymbol{E}}^{\mathrm{comp}}=\dot{\boldsymbol{E}}^{\|}+\dot{\boldsymbol{E}}^{\perp}, \quad \dot{\boldsymbol{E}}^{\|}=\sum_{i=1}^{3} a^{(i)} \boldsymbol{V}_{i}, \quad \dot{\boldsymbol{E}}^{\perp} \cdot \boldsymbol{V}_{i}=0 \quad i=1,2,3 .
$$

The deviation from overall isotropy is measured by :

$$
\eta=\frac{\left\|\dot{\boldsymbol{E}}^{\perp}\right\|}{\left\|\dot{\boldsymbol{E}}^{\text {comp }}\right\|}
$$

where

$$
\left\|\dot{\boldsymbol{E}}^{\mathrm{comp}}\right\|^{2}=\dot{\boldsymbol{E}}^{\mathrm{comp}}: \dot{\boldsymbol{E}}^{\mathrm{comp}}, \quad\left\|\dot{\boldsymbol{E}}^{\perp}\right\|^{2}=\left\|\dot{\boldsymbol{E}}^{\mathrm{comp}}\right\|^{2}-\sum_{i=1}^{3} \frac{\left(\dot{\boldsymbol{E}}^{\mathrm{comp}}: \boldsymbol{V}_{i}\right)^{2}}{\left\|\boldsymbol{V}_{i}\right\|^{2}} .
$$

Different values of $\eta$ found for the different configurations used in the present study can be found in Appendix C. It can be observed that the configurations, taken individually, may deviate significantly from isotropy (a few percents). However, when averages over several configurations are taken, the deviation from isotropy is small (less than $3.5 \%$ ). 
Remark: The differentiability of $\mathcal{F}$ used in (3) plays apparently an essential role in the proposed criterion (7). If the flow surface has a corner for a certain stress state, the direction of the strain-rate is non unique at this point. However, the deviation from isotropy can still be measured by (7) when the flow effective surface can be described by $N$ yield functions $\mathcal{F}_{i}$, all being isotropic and differentiable. Indeed, the flow rule takes the form

$$
\dot{\boldsymbol{E}}=\sum_{i=1}^{N} \dot{\lambda}_{i} \frac{\partial \mathcal{F}_{i}}{\partial \boldsymbol{\Sigma}}\left(\Sigma_{m}, \Sigma_{e q}, J_{3}\right),
$$

and the argument leading to the decomposition (5), on which the measure (7) is based, still holds for each of the terms in (8) individually.

The question of the existence of corners is difficult to answer. As Benzerga and Leblond (2010) point out, corners may arise on the effective flow surface at some stress states due to the occurence of plastic localization (taking over the diffuse plastic fields postulated by Gurson's criterion). In our simulations, several points (twenty in most cases) on the effective flow surface were determined but no information on the smoothness of the surface can be inferred from these simulations. We observed that, at the volume fraction considered in the present analysis, the flow surface did not exhibit sharp corners (at least at first sight), with a possible exception on the hydrostatic axis. A possible interpretation is that the localization criterion of Benzerga is not reached at the porosity considered here (4\%). But we cannot be more affirmative.

In the specific example of plastic voided materials which is the objective of the present study, it is often found that the deviation measured by (7) is maximal at high stress triaxiality. A possible explanation for this large deviation is that the flow surface could have a corner point (as found for two-dimensional voided materials under plane strain conditions by Pastor and Ponte Castañeda, 2002), or that the curvature of the effective flow surface in the vicinity of the hydrostatic axis is large. Small numerical errors may lead to artificially high values of the deviation from isotropy in the vicinity of such corner points.

\section{Numerical determination of the effective flow surface}

\subsection{Definition and properties of the effective flow surface}

The effective flow surface of voided materials is a special case of the more general problem of the effective strength domain of a composite material. Consider a composite material whose constituents have a limited strength characterized by a strength domain $P(\boldsymbol{x})$ at point $\boldsymbol{x}$, where $P$ is a convex domain in stress space. The associated dissipation function $\varphi$ (also called the support function of $P$ ) is defined as

$$
\varphi(\boldsymbol{x}, \dot{\varepsilon})=\sup _{\boldsymbol{\tau} \in P(\boldsymbol{x})} \boldsymbol{\tau}: \dot{\boldsymbol{\varepsilon}}
$$

$\varphi$ is a convex function as soon as $P$ is a convex set. 
The effective strength domain of the composite is defined as (Suquet, 1983, 1987):

$$
\begin{aligned}
P^{h o m}= & \{\boldsymbol{\Sigma} \text { such that there exists } \boldsymbol{\sigma}(\boldsymbol{x}) \text { with }\langle\boldsymbol{\sigma}\rangle=\boldsymbol{\Sigma}, \\
& \boldsymbol{\sigma}(\boldsymbol{x}) \in P(\boldsymbol{x}) \text { for all } \boldsymbol{x} \text { in } V, \operatorname{div} \boldsymbol{\sigma}(\boldsymbol{x})=\mathbf{0}, \boldsymbol{\sigma} \cdot \boldsymbol{n}-\#\},
\end{aligned}
$$

where - \# denotes a field taking opposite values on opposite sides of the unit-cell $V$ and $\boldsymbol{n}$ is the outer unit normal vector on the boundary of the unit-cell. The above definition is appropriate for periodic media (or periodic boundary conditions) but more general boundary conditions can be considered (Suquet, 1987). The boundary of $P^{\text {hom }}$ defines the effective extremal or flow surface of the composite (Hill, 1967). This surface depends on the strength domain of the individual constituents, on their volume fraction and on their arrangement. The reader is referred to Suquet (1987), de Buhan and Taliercio (1991), Francescato and Pastor (1997) among others for a discussion of the general properties of $P^{\text {hom }}$ and its applications to specific situations.

It is worth noting that the definition of $P^{\text {hom }}$ does not assume any constitutive relation for the phases except the information that no stress state outside $P(\boldsymbol{x})$ can be physically sustained at point $\boldsymbol{x}$. In particular the constituents are not assumed to be elasto-plastic. The counterpart of this generality is that the effective strength domain is only a domain of potential strength in the vocabulary of Salençon (2013). The numerical determination of $P^{\text {hom }}$ can be performed either by means of the static definition (9) or by means of a kinematic characterization based on the effective potential $\Phi$ (Suquet, 1987):

$$
\Phi(\dot{\boldsymbol{E}})=\inf _{\boldsymbol{v} \in \mathcal{K} \#(\dot{\boldsymbol{E}})}\langle\varphi(\dot{\boldsymbol{\varepsilon}}(\boldsymbol{v}))\rangle_{V},
$$

where $\mathcal{K}^{\#}(\boldsymbol{E})=\{\boldsymbol{u}$ such that $\boldsymbol{\varepsilon}(\boldsymbol{u})=\boldsymbol{E}+\boldsymbol{\varepsilon}(\boldsymbol{v}), \boldsymbol{v}$ periodic $\}$. It can be shown that $\Phi$ is the support function of $P^{\text {hom }}$ :

$$
\Phi(\dot{\boldsymbol{E}})=\sup _{\boldsymbol{\Sigma} \in P^{\text {hom }}} \boldsymbol{\Sigma}: \dot{\boldsymbol{E}} .
$$

Accurate upper and lower bounds for $P^{\text {hom }}$ can be obtained by means of these two approaches, as illustrated in Pastor et al. (2009) where the effective flow surface of a hollow sphere is investigated. The kinematic approach (10) leads to an upper bound for the effective flow surface, whereas the static approach (9) leads to a lower bound. The second approach (10) has been extensively used in the first part of this study (Vincent et al., 2013) (the only difference was that the displacement was assumed to be linear on the boundary of $V$ to simplify calculations). The full-field simulations reported in the following allow for the exact determination of the flow surface from the interior (it is therefore a static approach), up to numerical round-off errors.

Although the effective strength domain can be defined without reference to plasticity, two results specific to elastic ideally-plastic constituents are worth recalling. They are classical in the context of limit loads and can be extended to the present context of the effective extremal surface of composites. 
1. The effective flow surface can be characterized as the overall stress states obtained as asymptotic limits of the average stress along specific loading paths, assuming that each individual constituent is elasto-plastic, obeying the normality rule with respect to the domain of plasticity $P(\boldsymbol{x})$ at point $\boldsymbol{x}$. The effective extremal surface does not depend on the loading paths which have been followed to determine these asymptotic states and does not depend on the (real or artificial) elasticity assigned to the constituents.

2. The extremal surface does not depend on the type of plasticity theory, incremental or deformation theory, used for the individual constituents (provided that normality holds).

The first result is well-known and for the reader's convenience a brief derivation of the second result is given in Appendix A.

\subsection{The FFT method}

The numerical method used in the full-field simulations is based on fast Fourier transforms (FFT). In its simplest version, introduced in Moulinec and Suquet $(1994,1998)$ it solves an integral equation iteratively. The rate of convergence of this iterative algorithm deteriorates when the contrast between the phases increases. This slow convergence of the basic algorithm for composite with high contrast has motivated the development of accelerated schemes (Eyre and Milton, 1999; Michel et al., 2000; Zeman et al., 2010; Brisard and Dormieux, 2010; Monchiet and Bonnet, 2012; Moulinec and Silva, 2013) for composites with high contrast between the phases. In the case of voided materials such as those considered here, the contrast is even infinite. The accelerated method used here is based on augmented Lagrangians (Michel et al., 2001) and belongs to a broader class of accelerated schemes as shown by Moulinec and Silva (2013).

The extremal surface of the voided material is determined here using the deformation theory of plasticity (which delivers the same extremal surface as the incremental theory). Each individual constituent is governed by a strain-energy function $\varphi(\boldsymbol{x}, \boldsymbol{\varepsilon})$ such that, for all $\boldsymbol{x}$ in $V$ :

$$
\boldsymbol{\sigma}(\boldsymbol{x})=\frac{\partial \varphi}{\partial \varepsilon}(\boldsymbol{x}, \boldsymbol{\varepsilon}) \text {. }
$$

The volume $V$, consisting of the matrix material and of the voids, is subjected to an average strain $\boldsymbol{E}$ and periodicity conditions are assumed on its boundary. To be more specific, the strain field $\boldsymbol{\varepsilon}(\boldsymbol{u})$ is split into its average $\boldsymbol{E}$ and a fluctuation term $\boldsymbol{\varepsilon}(\boldsymbol{v})$ such that $\boldsymbol{\varepsilon}(\boldsymbol{u})=$ $\boldsymbol{E}+\boldsymbol{\varepsilon}(\boldsymbol{v})$. The field $\boldsymbol{v}$ is periodic while the vector $\boldsymbol{\sigma} \cdot \boldsymbol{n}$ is anti-periodic on the boundary of $V$. The effective strain-energy can be characterized by the variational property (10). This variational problem is then formulated as a minimization problem under constraint:

$$
\inf _{\boldsymbol{e}} \inf _{\boldsymbol{u} \in \mathcal{K}^{\#}(\boldsymbol{E})}\langle\varphi(\boldsymbol{e})\rangle_{V} \text {, under the constraint: } \boldsymbol{\varepsilon}(\boldsymbol{u}(\boldsymbol{x}))-\boldsymbol{e}(\boldsymbol{x})=0 \quad \forall \boldsymbol{x} \in V .
$$

The augmented Lagrangian associated to the previous problem reads as:

$$
\mathcal{L}^{(0)}(\boldsymbol{\varepsilon}(\boldsymbol{u}), \boldsymbol{e}, \boldsymbol{\lambda})=\langle\varphi(\boldsymbol{e})\rangle_{V}+\langle\boldsymbol{\lambda}:(\boldsymbol{\varepsilon}(\boldsymbol{u})-\boldsymbol{e})\rangle_{V}+\frac{1}{2}\left\langle(\boldsymbol{\varepsilon}(\boldsymbol{u})-\boldsymbol{e}): \boldsymbol{C}^{(0)}:(\varepsilon(\boldsymbol{u})-\boldsymbol{e})\right\rangle_{V}
$$


The second order tensor $\boldsymbol{\lambda}$ is the Lagrange multiplier and the fourth order tensor $\boldsymbol{C}^{(0)}$ is the stiffness of the reference medium which must be appropriately chosen. The minimization problem under constraint can be re-written as a saddle-point problem

$$
\sup _{\boldsymbol{\lambda}} \inf _{\boldsymbol{e}} \inf _{\boldsymbol{u} \in \mathcal{K}^{\#}(\boldsymbol{E})} \mathcal{L}^{(0)}(\boldsymbol{\varepsilon}(\boldsymbol{u}), \boldsymbol{e}, \boldsymbol{\lambda})
$$

which can be solved using Uzawa's algorithm:

Iterate $i: \boldsymbol{e}^{i-1}$ and $\boldsymbol{\lambda}^{i-1}$ being given,

- step 1 : find $\boldsymbol{\varepsilon}\left(\boldsymbol{u}^{i}\right)$ solution of:

$$
\inf _{\boldsymbol{u} \in \mathcal{K}^{\#}(\boldsymbol{E})} \mathcal{L}^{(0)}\left(\boldsymbol{\varepsilon}(\boldsymbol{u}), \boldsymbol{e}^{i-1}, \boldsymbol{\lambda}^{i-1}\right),
$$

- step 2 : find $\boldsymbol{e}^{i}$ such that (for all $\boldsymbol{x}$ ),

$$
\frac{\partial \varphi}{\partial \boldsymbol{e}}\left(\boldsymbol{x}, \boldsymbol{e}^{i}\right)+\boldsymbol{C}^{(0)}: \boldsymbol{e}^{i}(\boldsymbol{x})=\boldsymbol{C}^{(0)}: \boldsymbol{\varepsilon}\left(\boldsymbol{u}^{i}(\boldsymbol{x})\right)+\boldsymbol{\lambda}^{i-1}(\boldsymbol{x})
$$

- step 3 : Update $\boldsymbol{\lambda}^{i}, \boldsymbol{\lambda}^{i}(\boldsymbol{x})=\boldsymbol{\lambda}^{i-1}(\boldsymbol{x})+\boldsymbol{D}^{(0)}:\left(\boldsymbol{\varepsilon}\left(\boldsymbol{u}^{i}(\boldsymbol{x})\right)-\boldsymbol{e}^{i}(\boldsymbol{x})\right)$.

$\boldsymbol{D}^{(0)}$ is a fourth-order tensor which gives the descent direction in the algorithm. Once convergence has been reached, $\boldsymbol{e}$ coincides with $\boldsymbol{\varepsilon}(\boldsymbol{u})$ and $\boldsymbol{\lambda}$ is the stress.

\subsubsection{Step 1}

Step 1 is a classical linear elasticity problem for a homogeneous linear elastic medium with stiffness $\boldsymbol{C}^{(0)}$ :

$$
\boldsymbol{\sigma}(\boldsymbol{x})=\boldsymbol{C}^{(0)}: \boldsymbol{\varepsilon}(\boldsymbol{v}(\boldsymbol{x}))+\boldsymbol{\tau}(\boldsymbol{x}), \quad \operatorname{div} \boldsymbol{\sigma}(\boldsymbol{x})=\mathbf{0} \quad \forall \boldsymbol{x} \in V, \quad \boldsymbol{v} \#, \quad \boldsymbol{\sigma} \cdot \boldsymbol{n}-\#,
$$

where the polarization field $\boldsymbol{\tau}(\boldsymbol{x})$ is defined as: $\boldsymbol{\tau}(\boldsymbol{x})=\boldsymbol{\lambda}^{i-1}(\boldsymbol{x})-\boldsymbol{C}^{(0)}: \boldsymbol{e}^{i-1}(\boldsymbol{x})+\boldsymbol{C}^{(0)}: \boldsymbol{E}$. The solution of problem (15) can be expressed by means of the periodic Green operator $\boldsymbol{\Gamma}^{(0)}$ associated with $\boldsymbol{C}^{(0)}$ :

$$
\boldsymbol{\varepsilon}(\boldsymbol{v}(\boldsymbol{x}))=-\boldsymbol{\Gamma}^{(0)} * \boldsymbol{\tau}(\boldsymbol{x}) \text { in real space, } \hat{\boldsymbol{\varepsilon}}(\boldsymbol{v}(\boldsymbol{\xi}))=-\hat{\boldsymbol{\Gamma}}^{0}(\boldsymbol{\xi}): \hat{\boldsymbol{\tau}}(\boldsymbol{\xi}) \text { in Fourier space, }
$$

where the symbol $*$ denotes the convolution product in real space. The Fourier transform $\hat{\boldsymbol{\Gamma}}^{(0)}$ being explicitly known for general $\boldsymbol{C}^{(0)}$ (Moulinec and Suquet, 1998), the solution of (15) can be conveniently computed in Fourier space through the second equation in (16) and then computed in real space using the inverse FFT.

\subsubsection{Step 2}

When the potential $\varphi$ is isotropic and can be written as a function of the first two invariants of $\varepsilon, \varphi(\varepsilon)=\varphi\left(\varepsilon_{m}, \varepsilon_{e q}\right)$ and when the reference medium is taken to be isotropic with bulk and shear moduli $k^{(0)}$ and $\mu^{(0)}$ respectively, the second step reduces to a set of two non linear equations:

$$
\left\{\begin{array}{l}
\frac{1}{3} \frac{\partial \varphi}{\partial \varepsilon_{m}}\left(e_{m}^{i}, e_{e q}^{i}\right)+3 k_{0} e_{m}^{i}=3 k_{0} \varepsilon_{m}\left(\boldsymbol{u}^{i}\right)+\boldsymbol{\lambda}_{m}^{i-1} \\
\frac{1}{3 \mu_{0}} \frac{\partial \varphi}{\partial \varepsilon_{e q}}\left(e_{m}^{i}, e_{e q}^{i}\right)+e_{e q}^{i}=\left(\varepsilon^{d}\left(\boldsymbol{u}^{i}\right)+\frac{1}{2 \mu_{0}}\left(\boldsymbol{\lambda}^{i-1}\right)^{d}\right)_{e q}
\end{array}\right.
$$




\subsubsection{Step 3}

The update of the Lagrange multiplier $\boldsymbol{\lambda}$ requires a specific choice of the fourth-order tensor $\boldsymbol{D}^{(0)}$. Among other possible choices, $\boldsymbol{D}^{(0)}$ was taken equal to $\boldsymbol{C}^{(0)}$ in the present work. This choice leads to $\boldsymbol{\lambda}^{i}=0$ in the voids.

\subsubsection{Convergence criterion}

The criterion adopted to stop the iterations reads as

$$
\frac{\left\|\varepsilon^{i}-\boldsymbol{e}^{i}\right\|}{\|\boldsymbol{E}\|} \leq \eta_{1}, \quad \text { and } \quad \frac{\left\|\boldsymbol{\lambda}^{i}-\frac{\partial \varphi}{\partial \varepsilon}\left(\varepsilon^{i}\right)\right\|}{\left\|\left\langle\boldsymbol{\lambda}^{i}\right\rangle_{V}\right\|} \leq \eta_{2}
$$

where $\eta_{1}$ and $\eta_{2}$ are two prescribed thresholds, with typically in our calculations, $\eta_{1}=\eta_{2}=$ $10^{-3}$.

\subsubsection{Deformation theory for Gurson matrix with internal pressure}

The effective flow surface is determined using a deformation theory for the matrix, which is a Gurson material with pressurized voids (in the original Gurson's criterion the voids are stress-free). It follows from Vincent et al. (2009) that the corresponding yield function reads as:

$$
f^{G u r}(\boldsymbol{\sigma})=\frac{\sigma_{e q}^{2}}{\sigma_{0}^{2}}+2 f_{b} \cosh \left(\frac{3}{2} \frac{\sigma_{m}+p_{b}}{\sigma_{0}}\right)-1-f_{b}^{2},
$$

where $f_{b}$ is the void volume fraction of the intraganular bubbles and $p_{b}$ is their internal pressure. The plastic strain derived from the normality rule (in the framework of a deformation theory) is:

$$
\varepsilon^{p}=\lambda^{p} \frac{\partial f^{G u r}}{\partial \boldsymbol{\sigma}}(\boldsymbol{\sigma})
$$

where $\lambda^{p}$ is the plastic multiplier. Assuming infinitesimal strains, a poroelastic strain is added to the plastic strain to form the total strain

$$
\varepsilon=\varepsilon^{e}+\varepsilon^{p}, \quad \varepsilon^{e}=\boldsymbol{C}^{-1}:\left(\boldsymbol{\sigma}+p_{b} \boldsymbol{i}\right) .
$$

For simplicity, the stiffness tensor $\boldsymbol{C}$ is assumed to be isotropic, with bulk and shear moduli $k$ and $\mu$. Finally the complete set of constitutive relations for the deformation theory of a pressurized Gurson matrix reads as:

$$
\left\{\begin{array}{l}
\varepsilon_{m}=\frac{\sigma_{m}+p_{b}}{3 k}+\lambda^{p} \frac{f_{b}}{\sigma_{0}} \sinh \left(\frac{3}{2} \frac{\sigma_{m}+p_{b}}{\sigma_{0}}\right) \\
\varepsilon_{e q}=\frac{\sigma_{e q}}{3 \mu}+2 \lambda^{p} \frac{\sigma_{e q}}{\sigma_{0}^{2}} \\
\lambda^{p} \geq 0, \quad f^{G u r}(\boldsymbol{\sigma}) \leq 0, \quad \lambda^{p} f^{G u r}(\boldsymbol{\sigma})=0 .
\end{array}\right.
$$

The augmented Lagrangian method requires, in step 2, the inverse relation, i.e. the relation giving $\boldsymbol{\sigma}=\frac{\partial \varphi}{\partial \boldsymbol{\varepsilon}}(\boldsymbol{\varepsilon})$ as a function of $\boldsymbol{\varepsilon}$. The relations (22) can inverted as follows: 
- Compute the trial stress $\boldsymbol{\sigma}^{T}=\boldsymbol{C}: \boldsymbol{\varepsilon}-p_{b} \boldsymbol{i}$, i.e. $\sigma_{m}^{T}=3 k \varepsilon_{m}-p_{b}, \quad \sigma_{e q}^{T}=3 \mu \varepsilon_{e q}$.

- If $f^{G u r}\left(\boldsymbol{\sigma}^{T}\right)<0$, then $\boldsymbol{\sigma}=\boldsymbol{\sigma}^{T}$.

- Otherwise, $\left(\sigma_{m}, \sigma_{e q}\right)$ are determined by solving $(22)$. The last line is replaced by the single equation $f^{\text {Gur }}\left(\sigma_{m}, \sigma_{e q}\right)=0$. The resulting set of nonlinear equations is solved for $\left(\sigma_{m}, \sigma_{e q}, \lambda^{p}\right)$ by means of the Newton-Raphson method. Finally, $\boldsymbol{\sigma}=\sigma_{m} \boldsymbol{i}+\boldsymbol{\sigma}^{d}$, with:

$$
\boldsymbol{\sigma}^{d}=\frac{2 \mu}{1+6 \frac{\lambda^{p} \mu}{\sigma_{0}^{2}}} \varepsilon^{d}
$$

\subsubsection{Strain or stress control}

The elasto-plastic problem can be solved either following a path in strain space or a path in stress space (Marigo et al., 1987; Suquet, 1987; Swan, 1994)). In the strain-controlled method, the strain-rate $\dot{\boldsymbol{E}}$ is prescribed and the overall stress $\boldsymbol{\Sigma}(t)$ is computed as the average of the local stress field. Then, for voided materials, $\boldsymbol{\sigma}(t, \boldsymbol{x})$ has a limit when $t$ tends to $+\infty$ ( $P^{\text {hom }}$ is bounded in all directions) denoted $\boldsymbol{\sigma}^{\infty}(\boldsymbol{x})$. It is readily seen from Appendix A that the associated macroscopic stress $\boldsymbol{\Sigma}^{\infty}$ is such that

$$
\dot{\boldsymbol{E}}:\left(\hat{\boldsymbol{\Sigma}}-\boldsymbol{\Sigma}^{\infty}\right) \leq 0, \quad \forall \hat{\boldsymbol{\Sigma}} \in P^{h o m} .
$$

Therefore, $\boldsymbol{\Sigma}^{\infty}$ is on the boundary of $P^{h o m}$ and $\dot{\boldsymbol{E}}$ is an outer normal to $P^{\text {hom }}$ at $\boldsymbol{\Sigma}^{\infty}$.

In practice it is often required, and more physically sound, to impose the direction of the overall stress, rather than the overall strain-rate, motivating the introduction of a stress-controlled method where $\boldsymbol{\Sigma}(t)$ is prescribed. However, as is well-known, imposing a prescribed path in stress space may lead to numerical instabilities when approaching the limit load (asymptotic stress). A mixed procedure is adopted in which only the direction $\Sigma^{0}$ of the overall stress is prescribed together with the strain-rate in this direction:

$$
\boldsymbol{\Sigma}(t)=h(t) \Sigma^{0}, \quad \boldsymbol{\Sigma}^{0}: \dot{\boldsymbol{E}}(t)=t .
$$

where the amplitude $h(t)$ is an unknown of the problem which is determined by prescribing the strain-rate in the imposed direction of overall stress. In the example of uniaxial tension in direction 3 (for instance), the procedure (25) amounts to imposing to the macroscopic stress to have only one nonvanishing component $\Sigma_{33}$ in the tensile direction, whereas the loading is applied by controlling the strain $E_{33}$. This procedure can be seen as a strain-controlled loading in a prescribed direction only. When $t$ goes to $+\infty, h(t)$ and $\dot{\boldsymbol{E}}(t)$ have limits $h^{\infty}$ and $\dot{\boldsymbol{E}}^{\infty}$ when $t$ tends to $+\infty$ and these limits satisfy (24). The stationary stress $h^{\infty} \boldsymbol{\Sigma}^{0}$ is on the boundary of $P^{\text {hom }}$ with outer normal $\dot{\boldsymbol{E}}^{\infty}$. An additional advantage of controlling the direction of overall stress is that calculations can be performed at a prescribed macroscopic stress triaxiality-ratio $T$ :

$$
T=\frac{\Sigma_{m}}{\Sigma_{e q}}
$$


In principle, the calculations should be performed for a prescribed value of the differential pressure $p=p_{b}-p_{e}$. However convergence issues were observed if one tries to impose a significant internal pressure in the voids. This is probably due to the fact that the radial loading path in stress space starts from 0 , whereas the effective flow surface is translated along the hydrostatic axis. Therefore, depending on the stress triaxiality ratio, the boundary of the effective flow stress can be close to 0 . To avoid these convergence issues, the internal pressure has been applied gradually.

\subsection{Overall stress states and loading conditions}

The overall stress states considered in this study are taken in the following form

$$
\boldsymbol{\Sigma}=\Sigma_{m} \boldsymbol{i}+\frac{2}{3} \Sigma_{e q}\left(\begin{array}{ccc}
-\cos \left(\omega+\frac{\pi}{3}\right) & 0 & 0 \\
0 & -\cos \left(\omega-\frac{\pi}{3}\right) & 0 \\
0 & 0 & \cos \omega
\end{array}\right)
$$

The Lode angle $\omega$ varies from 0 to $\pi / 3$ and is related to the third invariant of the overall stress through

$$
J_{3}=\operatorname{det}\left(\Sigma^{d}\right)=\frac{2}{27} \Sigma_{e q}^{3} \cos (3 \omega) .
$$

Two specific values of the Lode angle, $\omega=0$ and $\omega=\pi / 6$, corresponding respectively to stress deviator $\Sigma^{d}$ being a purely axisymmetric, traceless stress state, to an in-plane shear, will be considered in the sequel:

$$
\left.\begin{array}{ll}
\omega=0: \quad \boldsymbol{\Sigma}^{d}=\frac{2}{3} \Sigma_{e q}\left(-\frac{1}{2} \boldsymbol{e}_{1} \otimes \boldsymbol{e}_{1}-\frac{1}{2} \boldsymbol{e}_{2} \otimes \boldsymbol{e}_{2}+\boldsymbol{e}_{3} \otimes \boldsymbol{e}_{3}\right), \\
\omega=\frac{\pi}{6}: \quad \boldsymbol{\Sigma}^{d}=\frac{1}{\sqrt{3}} \Sigma_{e q}\left(-\boldsymbol{e}_{2} \otimes \boldsymbol{e}_{2}+\boldsymbol{e}_{3} \otimes \boldsymbol{e}_{3}\right) .
\end{array}\right\}
$$

\section{Full-field simulations versus analytical model.}

\subsection{Preliminaries}

\subsubsection{Comparison with analytical models: modified porosity}

In order for the comparison between full-field simulations and the analytical models developed in part I of this study to be meaningful, a consistent definition of porosity has to be adopted on both sides. This is the role played in the GTN model by the parameter $q_{1}$. Following Tvergaard (1982), if $f$ is the void volume fraction in the axisymmetric unit-cell used in the full-field simulations, the results of these simulations should be compared to the Gurson model with a modified porosity $q_{1} f$, where $q_{1}$ ranges approximately from 1.15 to 1.7. The need for this correction can be understood at large volume fraction. The unit-cell loses its carrying capacity when the spherical void reaches the boundary of the cylinder, which occurs when $f=2 / 3$, leading to $q_{1}=1.5$, the value proposed by Tvergaard (1982) for small $f$, based on an analysis of strain localization between voids. Koplik and Needleman (1988) found a good agreement between their unit-cell simulations and Gurson criterion with $q_{1}=1.25$ halfway between the original Gurson's value $\left(q_{1}=1\right)$ and Tvergaard's value $\left(q_{1}=1.5\right)$. Other authors (Fritzen et al., 2012) have found a dependence of $q_{1}$ on $f$, in the 
form $q_{1}=1.69-f$. Despite the diversity in these expressions, it is clear that a correction must be applied to the porosity used in the simulations. In the present context, this correction applies to the intergranular porosity $f_{e}$ and the porosity $f_{e}^{*}$ used in the analytical models is defined with a correcting factor $q$ :

$$
f_{e}^{*}=q f_{e}
$$

The strategy adopted here to determine an estimate of $q$ follows closely the procedure through which the microstructures are constructed, by packing spheres in a first step (the packing volume fraction is denoted by $d$ ) and then by adding voids at the center of these spheres. The meaningful porosity $f^{*}$ used in the analytical model is the porosity of the ellipsoidal void in the ellipsoidal volume of matrix confocal to the void (see Figure 5). Let $a_{1}$ and $b_{1}$ denote the short and long axes of the spheroidal voids and $a_{2}$ and $b_{2}$ denote the axes of the confocal volume of matrix. Then the following relations can be easily established:

$$
f^{*}=\frac{a_{1} b_{1}^{2}}{a_{2} b_{2}^{2}}, \quad \frac{f_{e}}{d}=\frac{w b_{1}^{3}}{b_{2}^{3}}, \quad b_{1}^{2}-a_{1}^{2}=b_{2}^{2}-a_{2}^{2},
$$

from which the following relation is derived

$$
f^{*}=\left(1-\left(\frac{f_{e}}{w d}\right)^{2 / 3}\left(1-w^{2}\right)\right)^{-1 / 2} \frac{f_{e}}{d} .
$$

This would suggest to take $q=(1 / d)\left(1-\left(\frac{f_{e}}{w d}\right)^{2 / 3}\left(1-w^{2}\right)\right)^{-1 / 2}$. To be consistent with Koplik and Needleman (1988) we follow their suggestion to take $q$ halfway between $q=1$ (when no correction due to the packing volume fraction $d$ is applied) and the above value. This leads to

$$
q=\frac{1}{2}\left(1+\frac{1}{d\left(1-\left(\frac{f_{e}}{w d}\right)^{2 / 3}\left(1-w^{2}\right)\right)^{1 / 2}}\right) .
$$

Note that for spherical voids $(w=1)$ the correction still depends on the volume fraction of the safety spheres in the unit-cell.

\subsubsection{Reference medium used in the full-field simulations}

The artificial elasticity attributed to all matrix materials used in this section, and the reference medium used in the FFT simulations (denoted with a superscript 0) are both isotropic with Young modulus and Poisson ratio chosen as

$$
E=10 \sigma_{0}, \quad E^{(0)}=7.5 \sigma_{0}, \quad \nu=\nu^{(0)}=0.2,
$$

where $\sigma_{0}$ is the yield stress of the von Mises matrix at the microscopic scale.

\subsection{Spherical intergranular voids}

The intergranular voids considered in this subsection are spherical and all different microstructures have the same void volume-fraction $f_{e}=0.04$. 

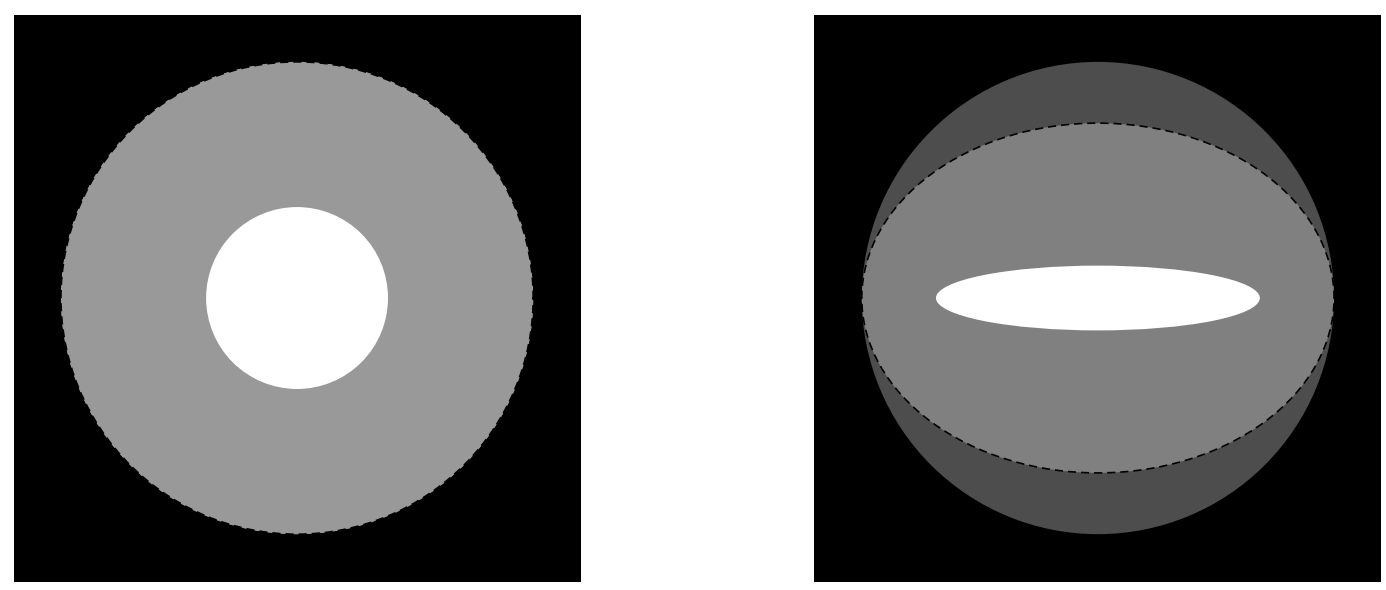

Figure 5: The porosity $f^{*}$ is defined as the ratio between the volume occupied by a void (in white) to the volume occupied by its confocal ellipsoid (in light gray) included in its outer sphere (in dark gray). Left: spherical (in this case, the confocal ellipsoid and the outer sphere are merged). Right: ellipsoidal in shape.

\subsubsection{Von Mises matrix}

As a preliminary test to the situation where two different populations of voids are present, the particular case of an incompressible von Mises matrix $\left(f_{b}=0\right)$ is investigated first. Since the matrix is incompressible, an internal pressure in the intergranular voids $\left(p_{e} \neq 0\right)$ results in a translation of the effective flow surface for the drained material $\left(p_{e}=0\right)$ along the hydrostatic axis. Therefore, the full-field simulations are performed with drained conditions only.

Six different realizations shown in Figure 6 containing 73 small, 12 medium, and 8 large voids were used in the full-field simulations (the size ratios with respect to the smallest size are respectively 1.0, 1.8, and 3.5). The filling rate $d$, defined as the ratio between the volume occupied by all single hollow spheres to the volume of the unit cell, is $d=0.7$. The resulting $q$ parameter, as defined in section 4.1.1 is therefore $q=1.214$. Each realization is discretized in $512^{3}$ voxels for the FFT simulations.

The two forms (28) of the overall stress have been considered in this study, corresponding to purely axisymmetric or purely in-plane shear $\boldsymbol{\Sigma}^{d}$. Each realization is loaded along 13 different directions corresponding to 13 different values of the stress triaxiality-ratio:

$$
T=-\infty,-20,-4,-2,-1,-1 / 3,0,1 / 3,1,2,4,20,+\infty
$$

and the limit stress in each direction is determined. As can be seen from Figures 7 and 8 the discrepancy between the 6 effective flow surfaces obtained for the 6 different configurations is small. Since the full-field simulations for the 6 different configurations are performed at the same stress triaxiality-ratios, the 6 results are aligned along radial directions in stress space. For each stress-triaxiality ratio, the discrepancy between the 6 configurations can be 


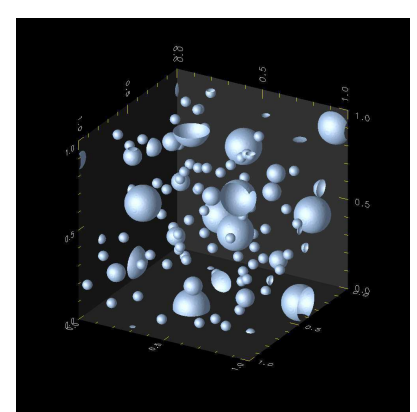

Microstructure \# 1

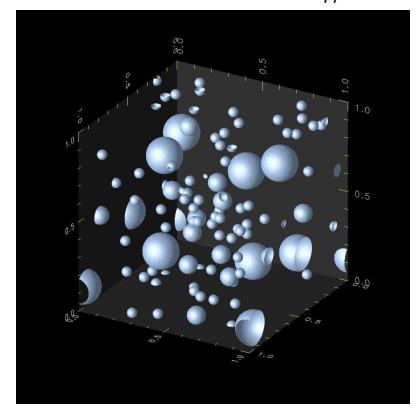

Microstructure \# 4

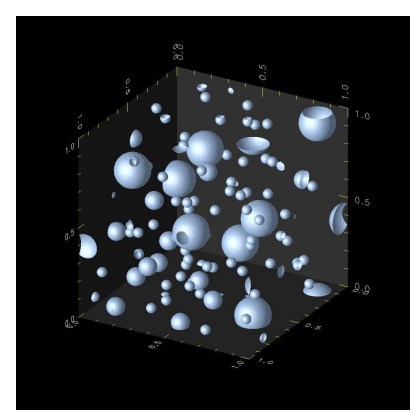

Microstructure \# 2

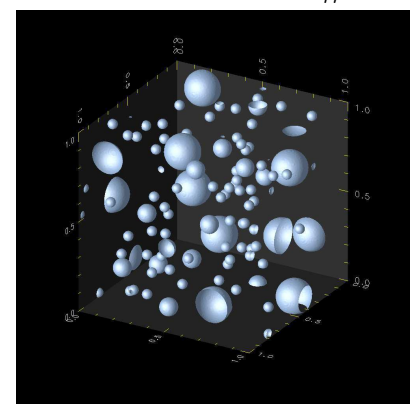

Microstructure \# 5

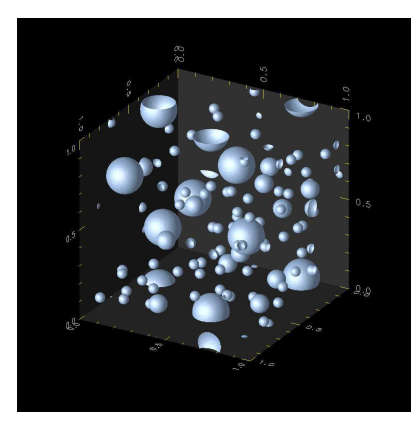

Microstructure \# 3

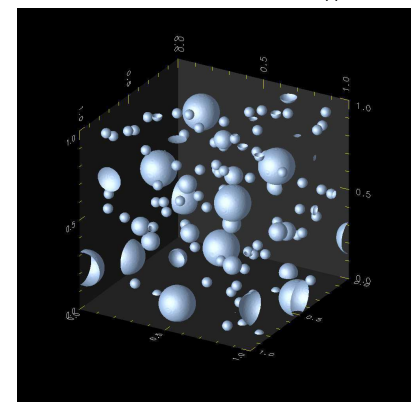

Microstructure \# 6

Figure 6: Spherical intergranular voids. Six different realizations of microstructures.

measured by the normalized root mean square:

$$
\epsilon=\frac{\sqrt{\sum_{k=1}^{n}\left(\left\|\Sigma^{(k)}\right\|-\|\bar{\Sigma}\|\right)^{2}}}{\sqrt{n}\|\bar{\Sigma}\|}
$$

where the superscript $k$ refers to the $k$-th realization, $n=6$ and

$$
\bar{\Sigma}=\frac{1}{n} \sum_{k=1}^{n} \Sigma^{(k)}, \quad\|\Sigma\|=\sqrt{\Sigma_{i j} \Sigma_{i j}}=\sqrt{3 \Sigma_{m}^{2}+\frac{2}{3} \Sigma_{e q}^{2}} .
$$

The error $\epsilon$ is plotted in Figures 9 and 10 for the 13 different stress triaxiality ratios. The error remains small (less than $0.7 \%$ ). The deviation from isotropy for the 6 different configurations and for their ensemble average is given in tables C.1 and C.2. Even though each configuration, taken individually, may deviate from isotropy by a few percents, the deviation obtained after averaging over the configurations is much smaller (less than $3.5 \%$ ). 


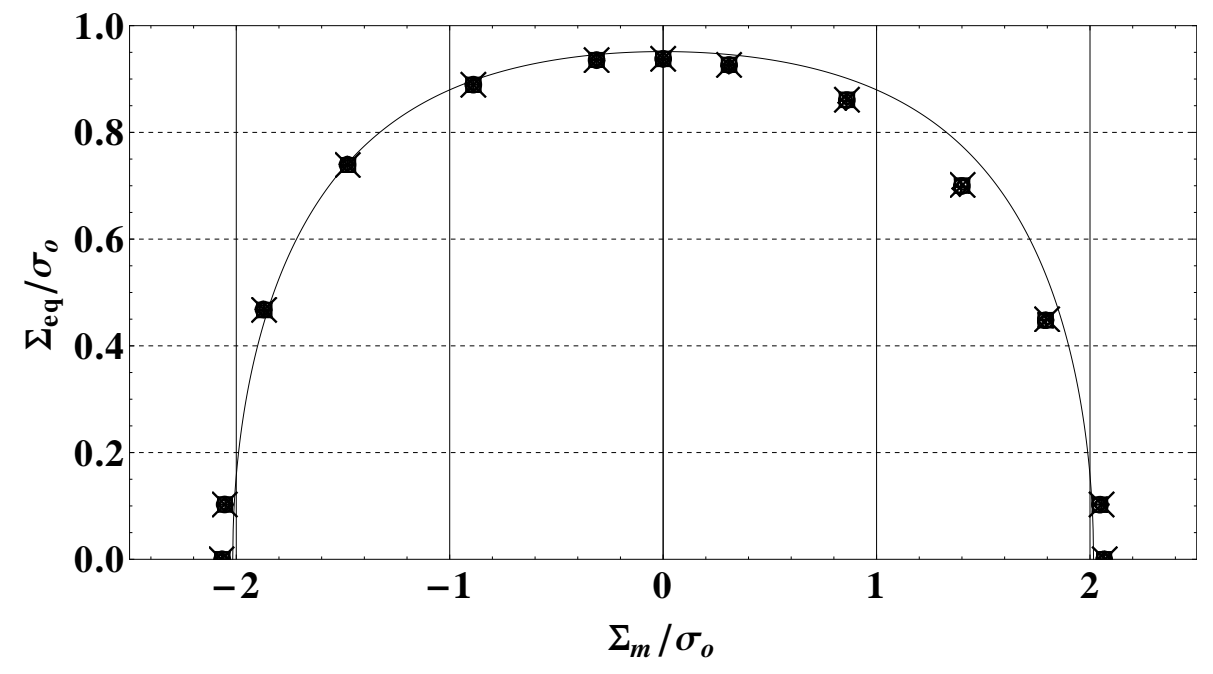

Figure 7: Spherical intergranular voids, von Mises matrix, Lode angle $\omega=0$, drained conditions $\left(p_{b}=\right.$ $p_{e}=0$ ). Solid line: Gurson criterion with porosity $q \times 0.04, q=1.214$. Symbols: FFT results for the 6 microstructures of Figure 6 (all 6 symbols are almost on top of each other).

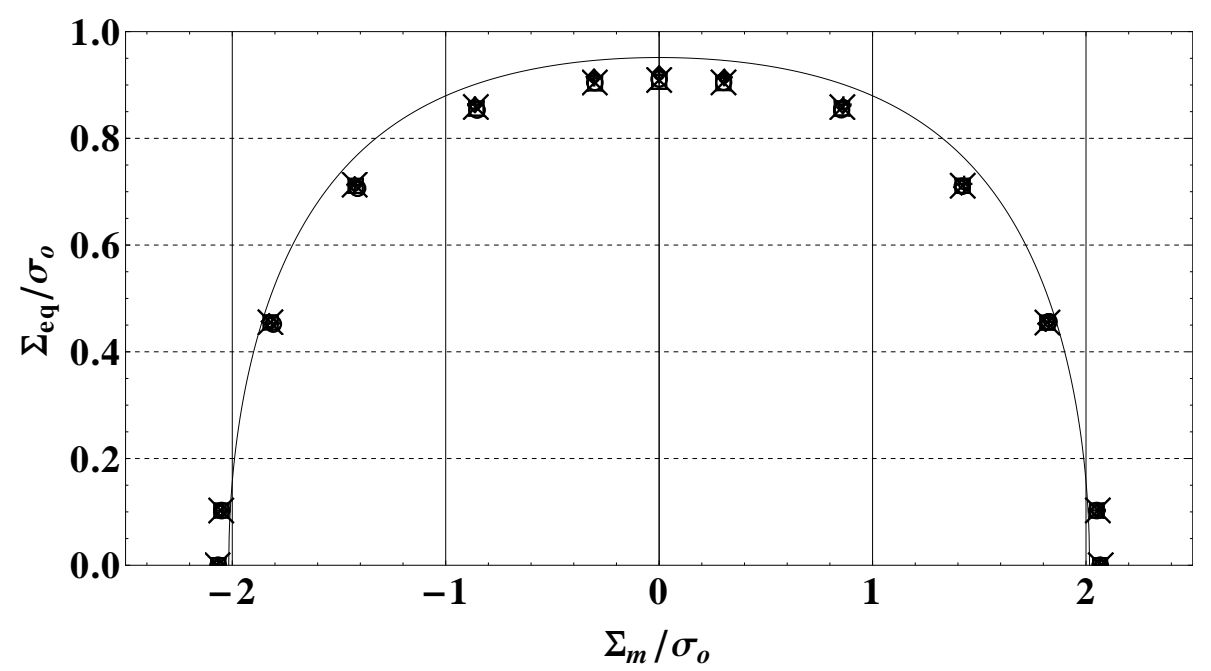

Figure 8: Spherical intergranular voids, von Mises matrix, Lode angle $\omega=\pi / 6$, drained conditions $\left(p_{b}=\right.$ $\left.p_{e}=0\right)$. Solid line: Gurson criterion with porosity $q \times 0.04, q=1.214$. Symbols: FFT results for the 6 microstructures of Figure 6 all 6 symbols are almost on top of each other). 


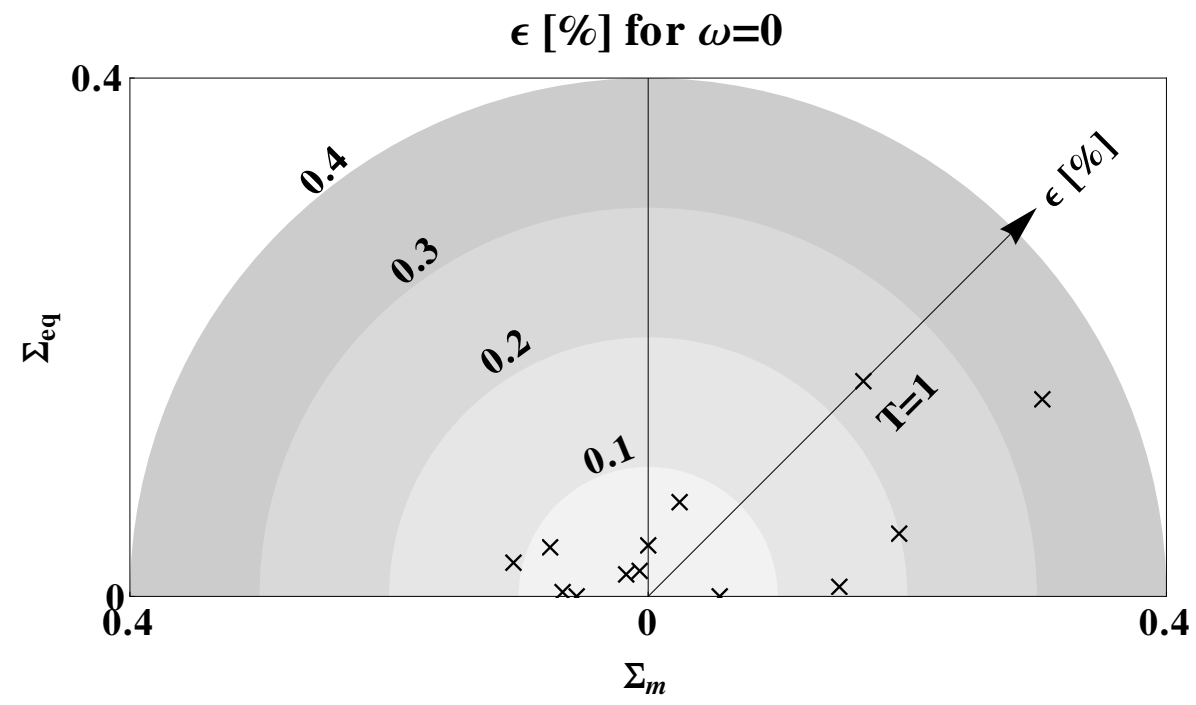

Figure 9: Spherical intergranular voids, von Mises matrix, Lode angle $\omega=0$, drained conditions $\left(p_{b}=p_{e}=\right.$ $0)$. Error $\epsilon$ as a function of the stress triaxiality-ratio (6 microstructures of Figure 6).

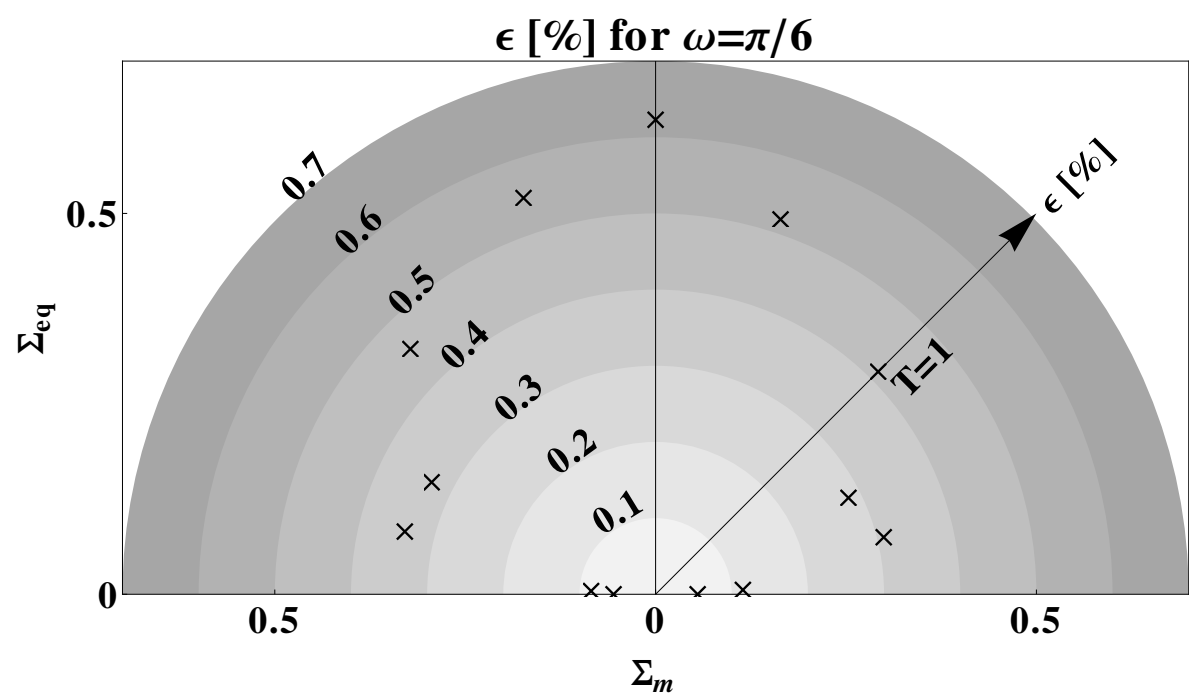

Figure 10: Spherical intergranular voids, von Mises matrix, Lode angle $\omega=\pi / 6$, drained conditions $\left(p_{b}=\right.$ $\left.p_{e}=0\right)$. Error $\epsilon$ as a function of the stress triaxiality-ratio (6 microstructures of Figure 6$)$. 


\subsubsection{Von Mises matrix, reduced configuration}

The small discrepancy observed among the 6 configurations of Figure 6 suggests that accurate results could be obtained with a single configuration. In addition, given the number of simulations which have to be performed at different internal pressures for a Gurson matrix, reducing the size of the configuration is desirable. This motivates the present section where full-field simulations are performed on a reduced configuration and compared to the reference results obtained in section 4.2.1. This reduced configuration shown in Figure 2 (right) contains 9 small, 3 medium, and 1 large voids. The spatial resolution of the image is $256^{3}$ voxels (compared to $512^{3}$ for the microstructures used in section 4.2.1). Typical computation times are 3 hours and 20 minutes for a microstructure with $256^{3}$ voxels and 41 hours for $512^{3}$ voxels (computations on 8 cores of a Hewlett-Packard Z800 workstation, equipped with two quadcore processors Intel ${ }^{\circledR}$ Xeon ${ }^{\circledR}$ W5580 3.20GHz).

The flow surface computed for this reduced configuration is compared, for axisymmetric stress states $(\omega=0)$, in Figure 11 to the classical Gurson criterion (with modified porosity $q f_{e}$ ) and with the average (for each direction of the stress) over the 6 configurations of the previous section. It can be observed that the three surfaces are in good agreement, which suggests that:

1. the correcting factor $q$ presented in section 4.1.1 leads to a good agreement between the analytical Gurson criterion and the full-field simulations (the flow stress under purely hydrostatic stress is slightly underestimated by Gurson's model, whereas the flow stress under purely deviatoric stress is slightly overestimated),

2. the effective flow surface can be determined with a good accuracy from full-field simulations on the reduced image only.

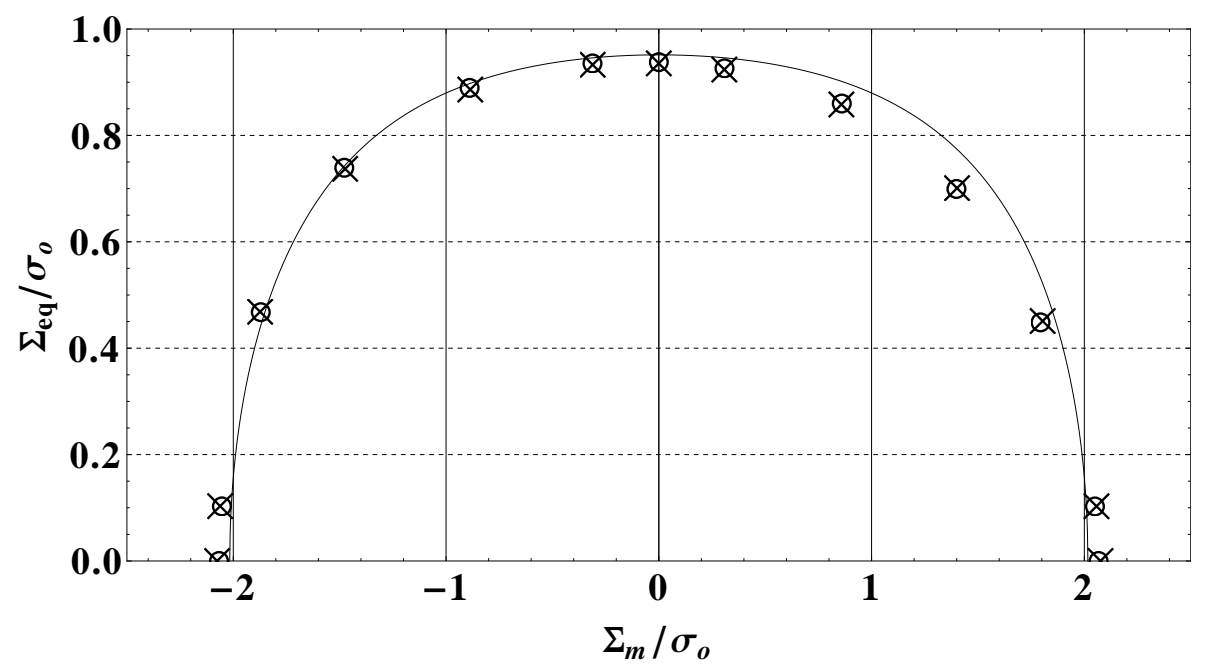

Figure 11: Spherical intergranular voids, von Mises matrix, Lode angle $\omega=0$, drained conditions $\left(p_{b}=\right.$ $\left.p_{e}=0\right)$. Solid line: Gurson criterion with porosity $q \times 0.04, q=1.214$. Crosses: FFT results on the reduced microstructure of Figure 2 (right). Circles: average over the 6 configurations of Figure 6 . 
The deviation from isotropy for this configuration is also reported in table C.1. The deviation for the reduced configuration is of the same order of magnitude as for the 6 configurations of the previous section: a few percents at high stress triaxiality and less than $1 \%$ at intermediate and low stress triaxiality.

Finally, given that the reduced configuration leads to almost the same results as the more elaborate configurations of Figure 6, all subsequent full-field simulations for spherical voids will be performed on the reduced configuration.

\subsubsection{Gurson matrix, reduced configuration}

The main objective of the present paper is to assess the accuracy of the analytical model (1) when both the primary and secondary populations of voids are present and subject to an internal pressure. For this purpose the matrix is now a Gurson material. The porosity of the secondary population (intragranular bubbles in the matrix) is $f_{b}=0.02$. The other parameters of the simulations are the same as in the previous section. Attention is restricted to axisymmetric overall stress states $(\omega=0)$. The effect of a differential pressure $p=p_{b}-p_{e}$ is investigated at three different values: $p_{b}=0, p_{b}=0.5 \sigma_{0}, p_{b}=1.5 \sigma_{0}$ ( $p_{e}$ is taken equal to $0)$. The comparison is performed at two levels:

1. Effective flow surface. The analytical criterion (1) is compared to the FFT results in Figure 12. The agreement is satisfactory for all three pressures at all stress triaxialities. The effect of an internal pressure $p_{b}$ is well reproduced by the model.

2. Average dilatation-rate in the matrix. The strain-rate field computed by the FFT simulations can be used to compare the average of the hydrostatic strain-rate in the matrix to the parameter $A$ introduced in the first part of the study. The results are shown in Figure 13 in the case of an overall hydrostatic loading. Additional values of the internal pressure $p_{b}$ have been considered here for the purpose of this comparison $\left(p_{b}=-1.0,-0.5,1.0,2.0,2.5\right)$. A good agreement between the model and the numerical simulations is observed which confirms that the expression $\bar{A}(p)$ proposed in Vincent et al. (2013) is an accurate approximation of the average dilatation-rate in the matrix. This is essential in studying the evolution of the void volume fraction of the intergranular voids which is governed by:

$$
\dot{f}_{e}=3\left(1-f_{e}\right)\left(\dot{E}_{m}-\left\langle\dot{\varepsilon}_{m}\right\rangle_{M}\right)
$$

An accurate estimate for $\left\langle\dot{\varepsilon}_{m}\right\rangle_{M}$ (the average dilatation rate in the matrix $M$ ) is required for a correct prediction of the evolution of $f_{e}$. As for the evolution of the intragranular void volume fraction $f_{b}$, it varies from point to point according to:

$$
\dot{f}_{b}(\boldsymbol{x})=3\left(1-f_{b}\right) \dot{\varepsilon}_{m}(\boldsymbol{x}), \quad \dot{f}_{b}(\boldsymbol{x})=3\left(1-f_{b}(\boldsymbol{x})\right) \dot{\varepsilon}_{m}(\boldsymbol{x}) .
$$

This relation can be averaged to give

$$
\overline{\dot{f}}_{b}=3\left(1-f_{b}\right)\left\langle\dot{\varepsilon}_{m}\right\rangle_{M},
$$




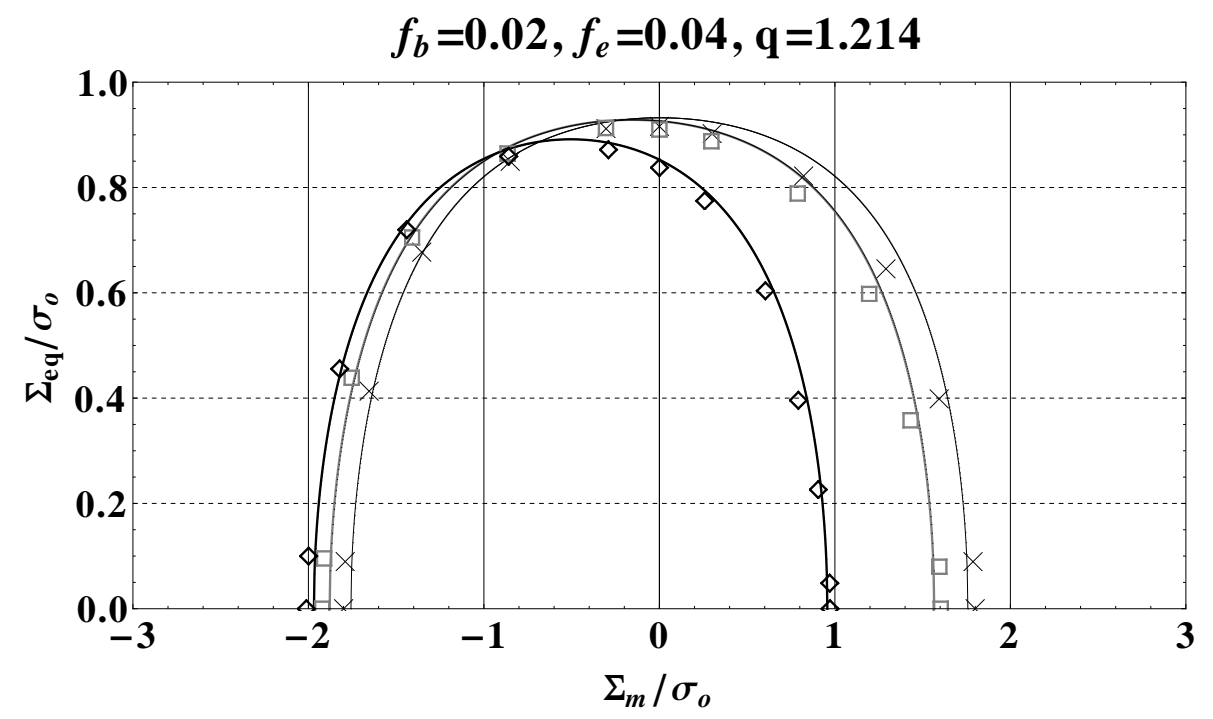

Figure 12: Spherical intergranular voids, Gurson matrix $f_{b}=0.02$, Lode angle $\omega=0$, microstructure Figure 2 (right): Solid line: new estimate (1) with $f_{e}^{*}=q \times 0.04, q=1.214, q_{3}=1, p_{e}=0$. Symbols: FFT simulations, Thin black: $p_{b}=0$. Gray: $p_{b}=0.5 \sigma_{0}$. Thick black: $p_{b}=1.5 \sigma_{0}$.

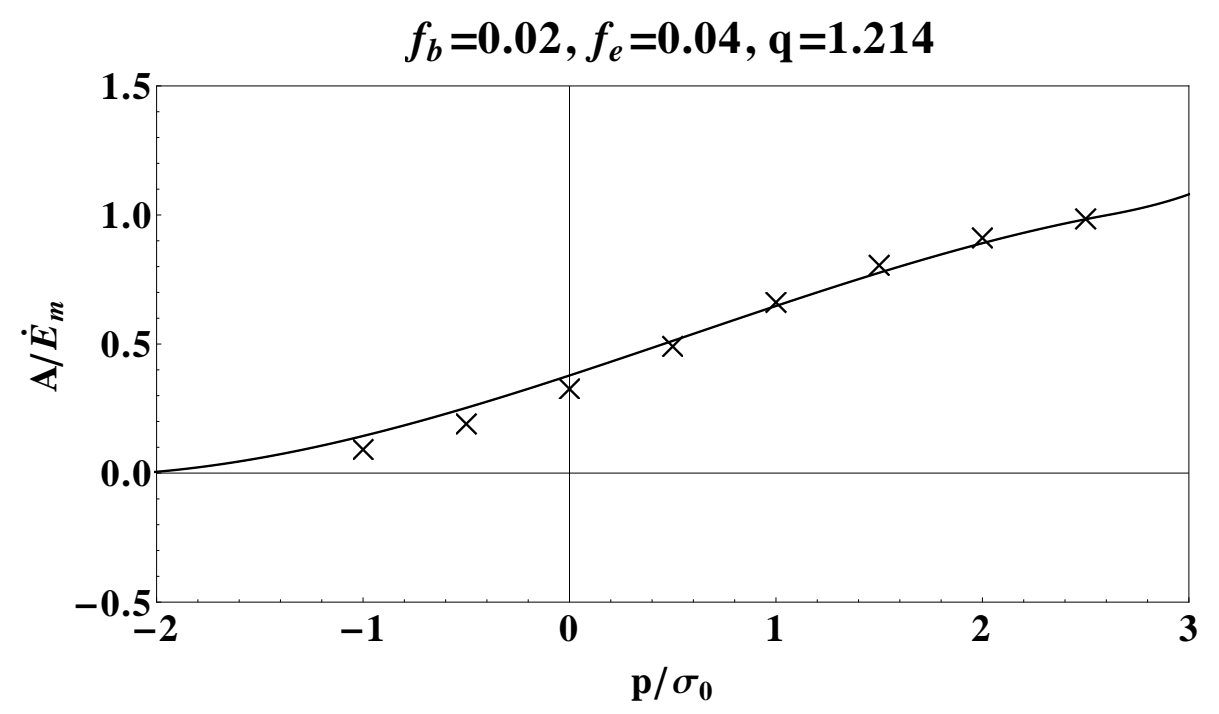

Figure 13: Spherical intergranular voids, Gurson matrix $f_{b}=0.02$, Lode angle $\omega=0$, microstructure Figure 2 (right). Hydrostatic loading. Average dilatation-rate in the matrix normalized by the average dilatation rate in the whole volume as function of $p=p_{b}-p_{e} \cdot p_{e}=0$. Solid line: estimate $\bar{A}$ proposed in Vincent et al. (2013) with $f_{e}^{*}=q \times 0.04, q=1.214, q_{3}=1$. Crosses: full-field simulations.

which can be seen as an evolution equation for the average microporosity. Again, an accurate estimate for $\left\langle\dot{\varepsilon}_{m}\right\rangle_{M}$ is essential for a correct prediction of the evolution (in average) of $f_{b}$.

The deviation from isotropy for the reduced configuration with a pressurized Gurson matrix is reported in table C.4. Although the deviation from isotropy depends on the 
constitutive relations of the matrix (and therefore depends on $f_{b}$ and $p_{b}$ ), it is found to be of the same order of magnitude as in the case of a Von Mises matrix.

\subsection{Ellipsoidal intergranular voids}

The intergranular voids considered in this section are ellipsoidal with aspect ratio $w=$ 1/5. Three different realizations, shown in Figure 3, have been used in the FFT simulations. They contain 511 ellipsoidal voids with a total volume fraction $f_{e}=0.04$, all with the same size but with random orientation. The unit-cell is discretized into $512^{3}$ voxels. The filling rate for the distribution of monodisperse spheres surrounding the ellipsoidal voids is $d=0.62$.

\subsubsection{Von Mises matrix}

As for spherical voids, the case of a von Mises matrix $\left(f_{b}=0\right)$ containing randomly oriented and distributed ellipsoidal voids (porosity $f_{e}$, aspect ratio $w$ ) is considered first. Three different microstructures (denoted by "microstructure \# 1" to "microstructure \# 3") have been generated following the procedure described in section 2.1. In a first step, 511 nonoverlapping spheres with identical size are arranged in the cubic unit-cell. The filling rate for 511 monodisperse spheres using our MD inspired algorithm is $d=0.62$. In a second step, ellipsoids (in gray in Figure 3) with identical size but different (random) orientation are located at the center of each individual sphere. Again, two Lode angles for the overall stress have been considered in this study $(\omega=0$ and $\omega=\pi / 6)$ corresponding to purely axisymmetric or purely in-plane shear $\Sigma^{d}$ (see section 3.3).

The effective flow surfaces obtained for these 3 different realizations are shown in figures 14 and 15 . The following observations can be made.

1. The discrepancy between the 3 realizations is small. This discrepancy can be estimated by the same measure (34) as in section 4.2.1, with $n=3$. The error $\epsilon$ is determined for each stress triaxiality ratio and shown in figures 16 and 17 . It is is less than $0.6 \%$, suggesting that stationarity has been reached with less than $1 \%$ error.

2. The agreement between the proposed analytical criterion (1) and the FFT results (figures 14 and 15) is good. The analytical model has been evaluated with the modified porosity $f_{e}^{*}=q f_{e}$ following the procedure of the section $4.1 .1(q=1.588)$. The GTN form of the effective flow surface is thus confirmed. The correcting factor $q$ of section 4.1.1 seems to match results at low and high stress triaxiality.

3. Finally, the deviation from isotropy has also been evaluated for the three configurations (Table C.3). Again, even though each configuration may deviate significantly from isotropy (up to $10 \%$ ), the deviation from isotropy of the ensemble average of the results (average over the 3 realizations) is small (less than $3.5 \%$ ). The deviation is maximal at high stress triaxiality, which suggests, as for spherical voids, that the curvature of the effective flow surface on the hydrostatic axis is large. 


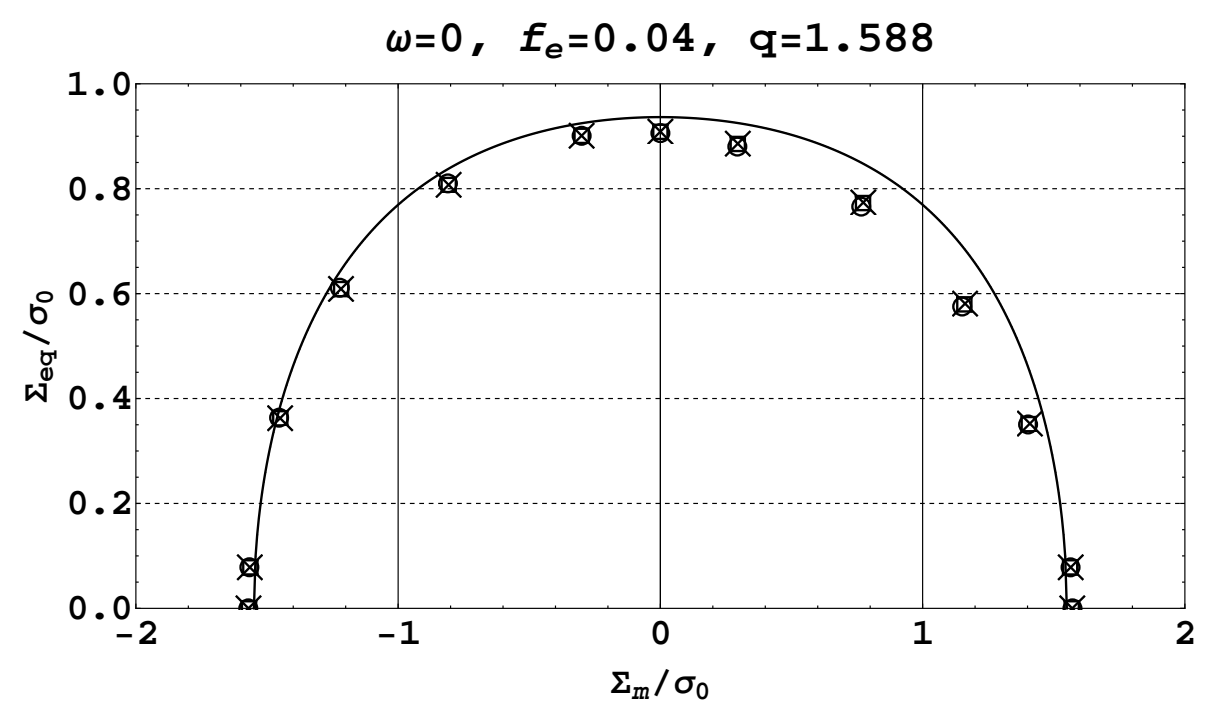

Figure 14: Ellipsoidal intergranular voids, von Mises matrix, Lode angle $\omega=0$, drained conditions $\left(p_{b}=\right.$ $\left.p_{e}=0\right)$. Microstructures \# 1 to \# 3 of Figure 3. Solid line: new estimate (1) with $f_{b}=0, f_{e}^{*}=q \times 0.04$, $q=1.588 w=1 / 5, q_{3}=1, p_{b}=p_{e}=0$. Symbols: FFT simulations.

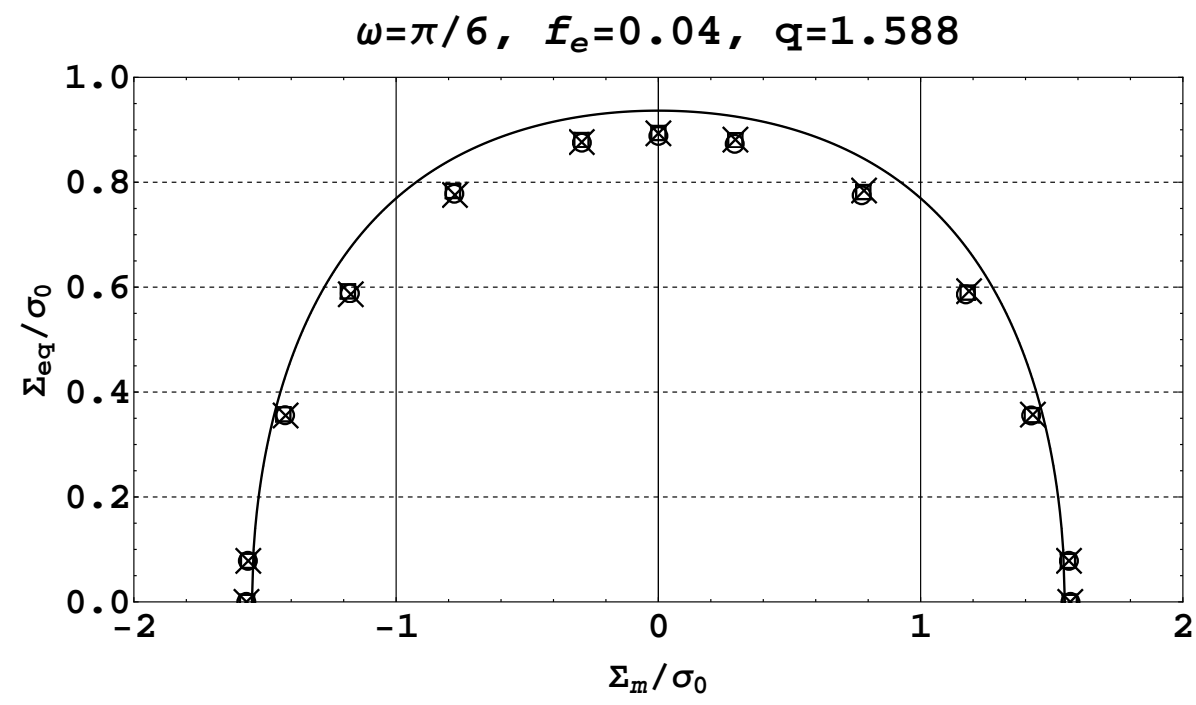

Figure 15: Ellipsoidal intergranular voids, von Mises matrix, Lode angle $\omega=\pi / 6$, drained conditions $\left(p_{b}=p_{e}=0\right)$. Microstructures \# 1 to \# 3 of Figure 3. Solid line: new estimate (1) with $f_{b}=0$, $f_{e}^{*}=q \times 0.04, q=1.588 w=1 / 5, q_{3}=1, p_{b}=p_{e}=0$. Symbols: FFT simulations.

\subsubsection{Gurson matrix}

Finally full-field simulations for a Gurson matrix $\left(f_{b} \neq 0\right)$ and different internal pressures $p_{b}$ in the ellipsoidal voids are compared with analytical model. Given the low dispersion of results among the 3 realizations, only microstructure \# 1 was investigated under axisymmetric overall stress $(\omega=0)$. 


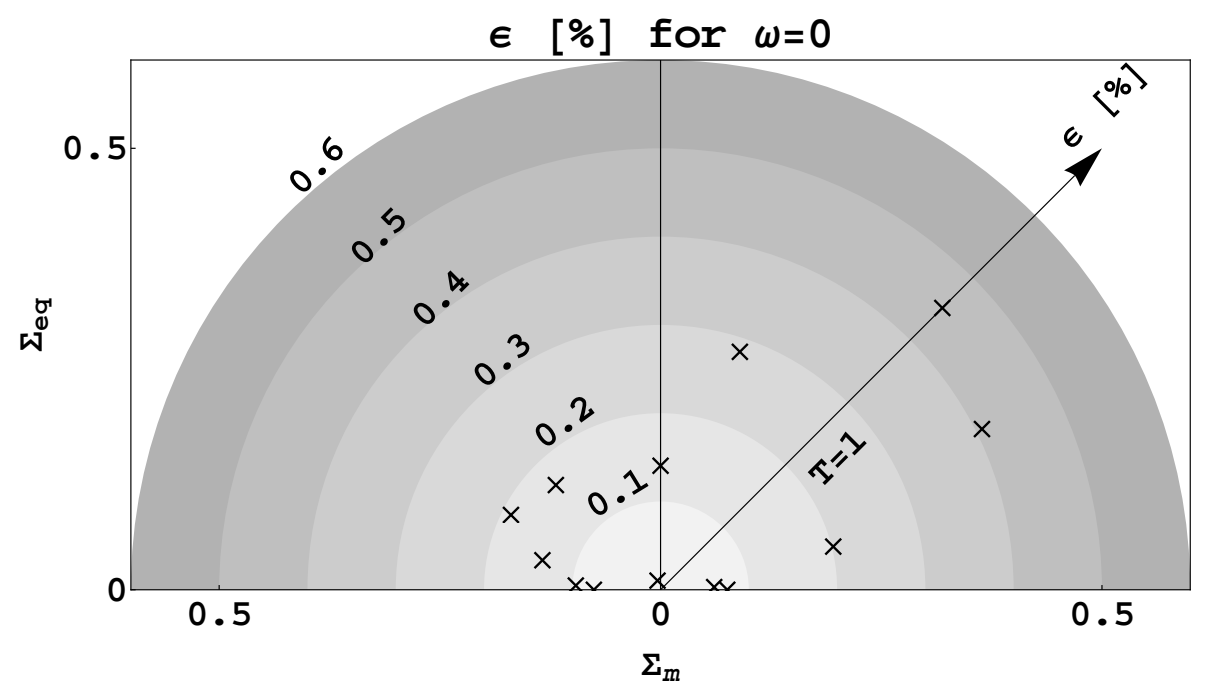

Figure 16: Ellipsoidal intergranular voids, von Mises matrix, Lode angle $\omega=0$, drained conditions $\left(p_{b}=\right.$ $\left.p_{e}=0\right)$. Error $\epsilon$ as a function of the stress triaxiality-ratio (3 microstructures of Figure 3).

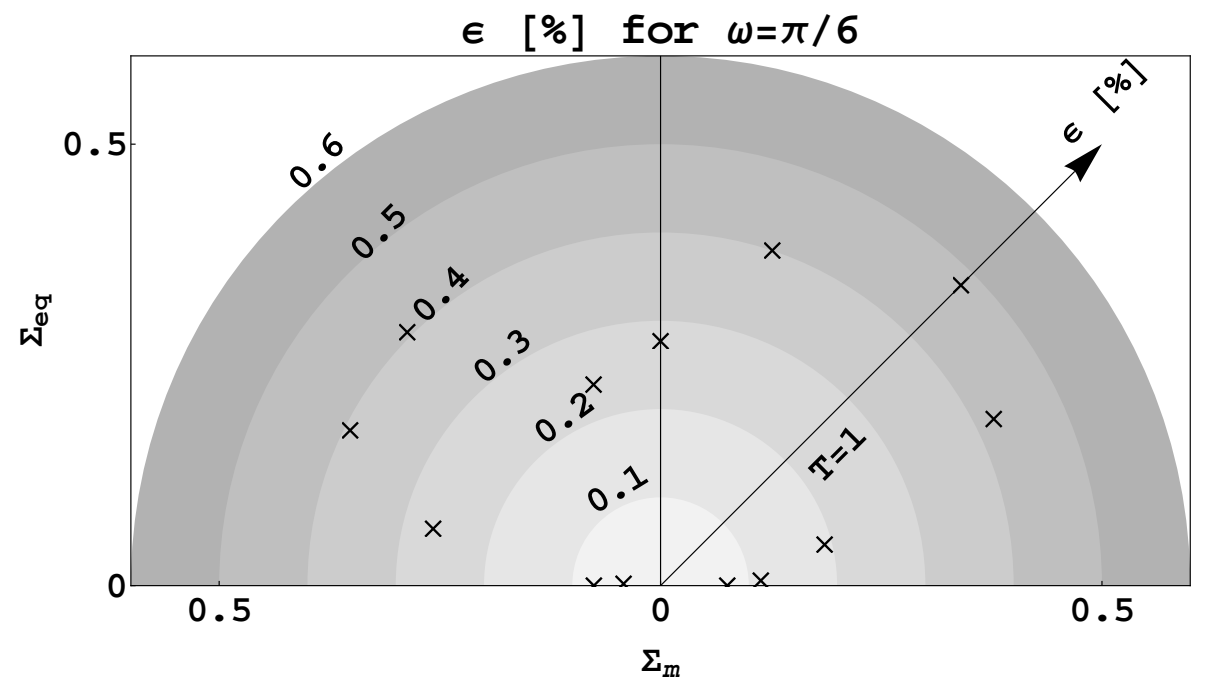

Figure 17: Ellipsoidal intergranular voids, von Mises matrix, Lode angle $\omega=\pi / 6$, drained conditions $\left(p_{b}=p_{e}=0\right)$. Error $\epsilon$ as a function of the stress triaxiality-ratio (3 microstructures of Figure 3$)$.

The agreement between the analytical criterion (1) and the FFT results is good for all values of the internal pressure $p_{b}$ which were considered in the simulations (see Figure 18). Moreover the average dilatation rate in the matrix with respect to the pressure $p_{b}$ can be computed from the full-field simulations and compared with the predictions of the approximation $\bar{A}$ introduced in the analytical model (Vincent et al., 2013). This is done in Figure 19 for a purely hydrostatic stress when $p_{b}=-1.0,-0.5,0,0.5,1.0,1.5,2.0,2.5$. Again, a good agreement between the model and the numerical simulations is observed. Once again the good agreement between the model and the full-field simulations, both for 
the effective flow surface and for the average dilatation-rate in the matrix (or equivalently with the void growth-rate) supports the claim that the effective flow surface of doubly porous materials with pressurized voids can be accurately approximated by a GTN-type criterion.

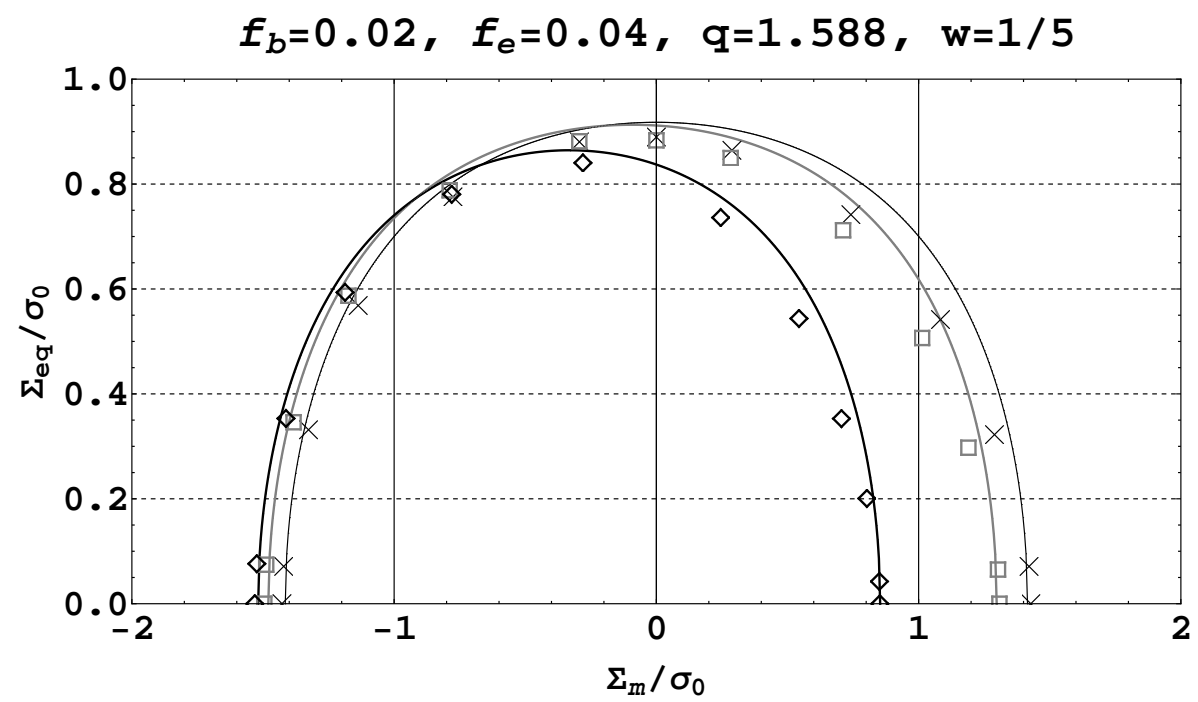

Figure 18: Ellipsoidal intergranular voids. Comparison between predictions for the effective flow surface. Lines: new estimate (1) computed with $f_{b}=0.02, f_{e}^{*}=q \times 0.04, q=1.588 w=1 / 5, q_{3}=1, p_{e}=0$. Symbols: FFT results on the microstructure \# 1 of the Figure 3. Thin black: $p_{b}=0$. Gray: $p_{b}=0.5 \sigma_{0}$. Thick black: $p_{b}=1.5 \sigma_{0}$.

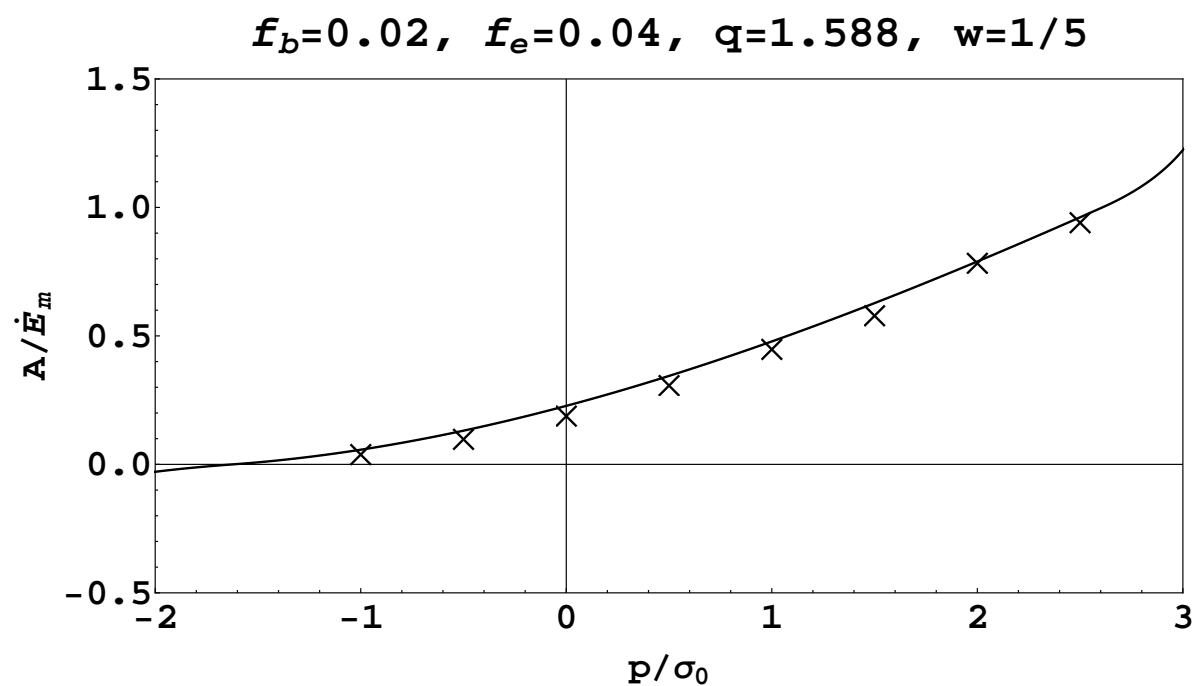

Figure 19: Ellipsoidal intergranular voids. Hydrostatic overall stress. Average dilatation-rate in the matrix normalized by the average dilatation rate in the whole volume function of $p$. Solid line: analytical model $\bar{A}$ of Vincent et al. (2013). Crosses: full-field simulations on microstructure \# 1 from the Figure 3.

The deviation from isotropy has also been evaluated (see table C.4). The results are 
roughly the same as in the case of a von Mises matrix with a maximum deviation at high triaxiality.

\subsection{Influence of the third invariant of the stress}

Although this study is not devoted to a thorough discussion of the influence of the third invariant of the overall stress on the plastic properties of voided materials, a few observations can be made from the full-field simulations. Indeed the analytical model (1) does not incorporate any dependence on the third invariant and the question of whether this approximation is relevant can be asked. We discuss here only the drained case $\left(p_{b}=p_{e}=0\right)$ for a von Mises matrix.

1. First the numerical effective flow surface for $\omega=0$ is not symmetric with respect to the deviatoric axis $\Sigma_{m}=0$, whereas it is symmetric when $\omega=\pi / 6$. This is consistent with the following exact result first proved in Cazacu et al. (2013): when the matrix has point symmetry (i.e. when the local plastic potential satisfies $\varphi(-\dot{\varepsilon})=\varphi(\dot{\varepsilon})$ ), the effective flow surface has also point symmetry (if $\boldsymbol{\Sigma}$ belongs to $P^{\text {hom }}$ then $-\boldsymbol{\Sigma}$ also belongs to $\left.P^{\text {hom }}\right)$. When the material has overall isotropy, the effective flow surface can be expressed in terms of the three first invariants of $\boldsymbol{\Sigma}$ and the point symmetry of $P^{\text {hom }}$ implies that if $\left(\Sigma_{m}, \Sigma_{e q}\right.$, $\left.\operatorname{det}(\boldsymbol{\Sigma})\right)$ belongs to $P^{\text {hom }}$ then $\left(-\Sigma_{m}, \Sigma_{e q},-\operatorname{det}(\boldsymbol{\Sigma})\right)$ also belongs to $P^{\text {hom }}$. This has two consequences:

- First, for a given Lode angle $\omega$, the two flow surfaces corresponding to $\omega$ and $\omega+\pi / 3$ in the plane $\left(\Sigma_{m}, \Sigma_{e q}\right)$ are deduced one from the other by a mirror symmetry with respect to the deviatoric axis. This is a consequence of the fact that when $\omega$ is changed into $\omega+\pi / 3, \operatorname{det}(\boldsymbol{\Sigma})$ is changed into $-\operatorname{det}(\boldsymbol{\Sigma})$.

- In particular when $\omega=\pi / 6$, i.e. when $\operatorname{det}(\boldsymbol{\Sigma})=0$, the corresponding effective flow surface has mirror symmetry with respect to effective flow surface

Therefore it is not surprising that the two flow surfaces for $\omega=\pi / 6$ in figures 8 and 15 are symmetric but that the two surfaces in figures 7 and 14 are asymmetric.

2. The asymmetry of the flow surfaces for $\omega=0$ is small. It cannot be concluded immediately that this small effect of the Lode's angle on the effective flow surfaces results in a small effect on the flow rule. As pointed out in Danas et al. (2008), the overall plastic strain-rate (colinear to the gradient to the flow surface) can be affected by a small perturbation of the surface, in particular in the vicinity of the hydrostatic axis where our numerical simulations show that the curvature of the surface may be large. However, a closer look at the overall dilatational strain-rates does not reveal a significant effect of the Lode's angle on the overall dilatation, at least in the range of porosity which was investigated here and this effect should not be overestimated.

3. However, a definite influence of the third invariant on the local plastic strain-rate fields was observed. In all our simulations, the fields corresponding to overall pure shear $(\omega=\pi / 6)$ were more localized (concentrated into highly deformed regions) than those obtained for axisymmetric shear $(\omega=0)$. This is illustrated in Figure 20 for instance with the following color code: red and blue correspond to high and low strainrate levels respectively, green and yellow to intermediate strain-rate levels (normalized 
by the overall strain-rate). Therefore, one cannot exclude that, integrated over the whole time history of the material, these small deviations in the flow rule and in the change of shape of voids may lead to significant differences at void coalescence for different Lode's angles.
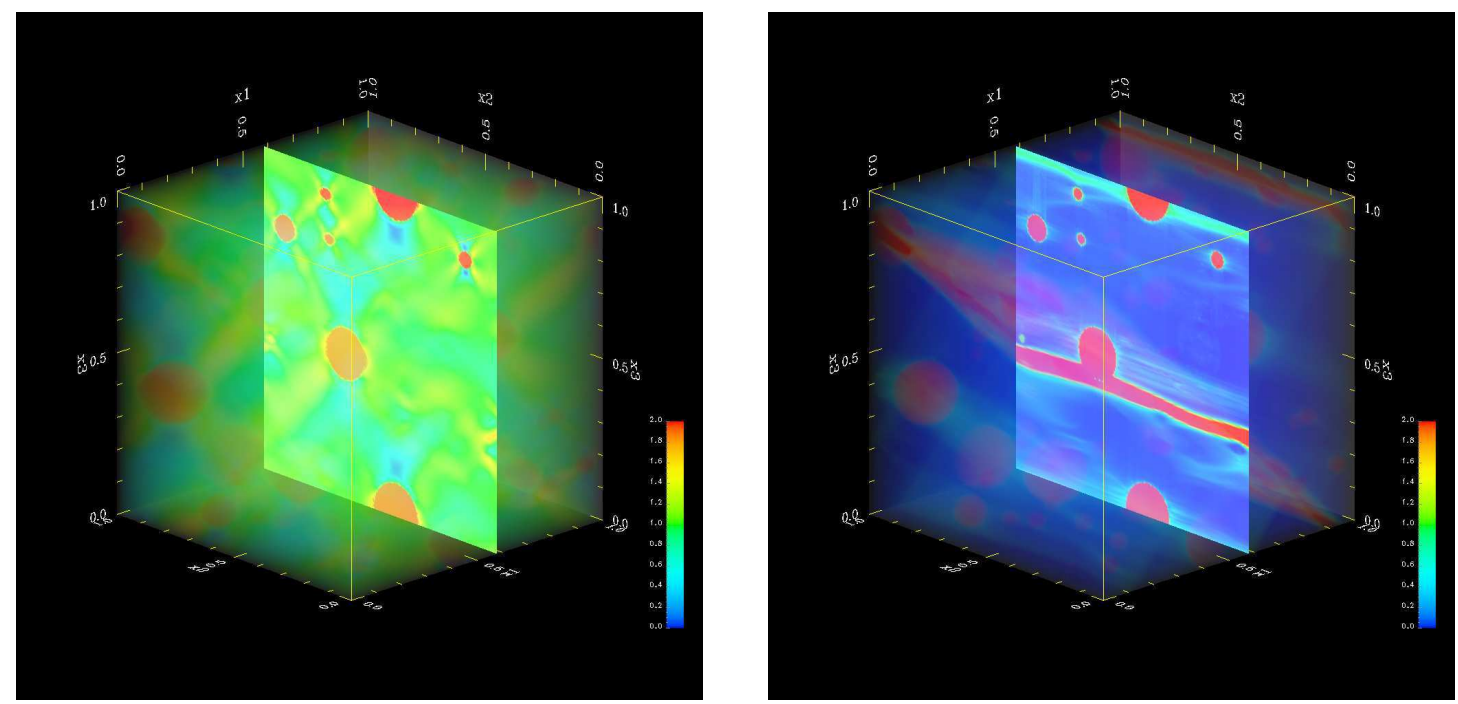

Figure 20: Spherical voids (microstructure \# 0 in Figure 6). Purely deviatoric overall stress. Snapshots of the equivalent strain-rate field. Left: $\omega=0$. Right: $\omega=\pi / 6$.

The consequences of this huge difference in the local strain-rate fields, which may have a significant effect on the change of shape of the voids and therefore on the evolution of the microstructure, remain to be investigated.

\section{General conclusion}

In part I of this study, an analytical model was proposed to describe the effective flow surface of a voided material with two populations of voids of different sizes, subjected to internal pressures in addition to the classical loading by average. In its simplest form, the analytical form which was proposed was of GTN-type. An explicit expression for the average dilatation-rate in the matrix (or alternatively of the void growth-rate) was also derived.

In the present paper (part 2 of the study), the predictions of this analytical criterion have been compared to full-field numerical simulations using the Fast Fourier Transform method of Moulinec and Suquet (1998) and Michel et al. (2000). The method has been successfully applied to the case of voided materials with a Gurson matrix and internal pressure. Different unit cells containing a rather large number of voids (511 voids in the ellipsoidal case) to ensure isotropy and representativeness up to a certain error (which has been defined) have been investigated. The agreement with the model is found to be very satisfactory. 
Acknowledments: H. Moulinec and P. Suquet acknowledge the financial support of Labex MEC and of A*Midex through grants ANR-11-LABX-0092 and ANR-11-IDEX-0001-02.

\section{References}

Benzerga, A. Leblond, J.B., 2010. Ductile fracture by void growth to coalescence. Advances in Applied Mechanics 44, 169-305.

Brisard, S., Dormieux, L., 2010. FFT-based methods for the mechanics of composites: A general variational framework. Computational Materials Science 49 (3), $663-671$.

Cazacu, O., Revil-Baudard, B., Lebensohn, R., Gărăjeu, M., 2013. On the combined effect of pressure and third invariant on yielding of porous solids with von mises matrix. Journal of Applied Mechanics , in press.

Danas, K., Idiart, M., Ponte Castañeda, P., 2008. A homogenization-based constitutive model for isotropic viscoplastic porous media. International Journal of Solids and Structures 45, 3392-3409.

de Buhan, P., Maghous, S., 1995. A straightforward numerical method for evaluating the ultimate loads of structures. Eur. J. Mech.: A/Solids 14, 309-328.

de Buhan, P., Taliercio, A., 1991. A homogenization approach to the yield strength of composites. Eur. J. Mech.: A/ Solids 10, 129-154.

Eyre, D., Milton, G., 1999. A fast numerical scheme for computing the response of composites using grid refinement. J. Physique III 6, 41-47.

Francescato, P., Pastor, J., 1997. Lower and upper numerical bounds to the off-axis strength of unidirectional fiber-reinforced composites by limit analysis methods. Eur. J. Mech., A/Solids 6, 213-234.

Fritzen, F., Forest, S., Bohlke, T., Kondo, D., Kanit, T., 2012. Computational homogenization of elastoplastic porous metals. International Journal of Plasticity 29, 102-119.

Fritzen, F., Forest, S., Kondo, D., Bòhlke, T., 2013. Computational homogenization of porous materials of Green type. Computational Mechanics 52, 121-134.

Gurson, A., 1977. Continuum theory of ductile rupture by void nucleation and growth: Part I - yield criteria and flow rules for porous ductile media. Journal of Engineering Materials and Technology 99, 1-15.

Hill, R., 1967. The essential structure of constitutive laws for metal composites and polycrystals. J. Mech. Phys. Solids 15, 79-95.

Kanit, T., Forest, S., Galliet, I., Mounoury, V., Jeulin, D., 2003. Determination of the size of the representative volume element for random composites: statistical and numerical approach. International Journal of Solids and Structures 40, 3647-3679.

Koplik, J., Needleman, A., 1988. Void growth and coalescence in porous plastic solids. International Journal of Solids and Structures 24, 835-853.

Lebensohn, R., Rollett, A., Suquet, P., 2011. Fast Fourier transform-based modelling for the determination of micromechanical fields in polycrystals. Journal of Materials $63,13-18$.

Marigo, J., Mialon, P., Michel, J., P.Suquet, 1987. Plasticité et homogénéisation : un exemple de prévision des charges limites d'une structure périodiquement hétérogène. J. Méca. Th. Appl. 6, 47-75.

Michel, J., Moulinec, H., Suquet, P., 2000. A Computational Method Based on Augmented Lagrangians and Fast Fourier Transforms for Composites with High Contrast. Computer Modeling in Engineering and Sciences 1 (2), 79-88.

Michel, J., Moulinec, H., Suquet, P., 2001. A computational method for linear and nonlinear composites with arbitrary phase contrast. Int. J. Numer. Meth. Engng 52, 139-160.

Monchiet, V., Bonnet, G., 2012. A polarization-based FFT iterative scheme for computing the effective properties of elastic composites with arbitrary contrast. Int. J. Numer. Meth. Engng 89, 1419-1436.

Moulinec, H., Silva, F., 2013. Comparison of three FFT-based iterative schemes for computing the mechanical response of composite materials. International Journal for Numerical Methods in Engineering, accepted.

Moulinec, H., Suquet, P., 1994. A fast numerical method for computing the linear and nonlinear properties of composites. C. R. Acad. Sc. Paris II 318, 1417-1423. 
Moulinec, H., Suquet, P., 1998. A numerical method for computing the overall response of nonlinear composites with complex microstructure. Computer Methods in Applied Mechanics and Engineering 157, 69-94.

Pastor, F., Loute, E., Pastor, J., Trillat, M., 2009. Mixed method and convex optimization for limit analysis of homogeneous Gurson materials: a kinematical approach. European Journal of Mechanics A/Solids 28, $25-35$.

Pastor, J., Ponte Castañeda, P., 2002. Yield criteria for porous media in plane strain: second-order estimates versus numerical results. C.R. Mecanique 330, 741-747.

Richelsen, A., Tvergaard, V., 1994. Dilatant plasticity or upper bounds estimates for porous ductile solids. Acta Metall. Mater. 42, 2561-2577.

Salençon, J., 2013. Yield Design. ISTE. John Wiley.

Stoyan, D., Kendall, W., Mecke, J., 1995. Stochastic Geometry and Its Applications. Wiley Series in Probability and Statistics. John Wiley \& Sons Ltd, England.

Suquet, P., 1983. Analyse limite et homogénéisation. C.R. Acad. Sc. Paris, II 296, 1355-1358.

Suquet, P., 1987. Elements of Homogenization for Inelastic Solid Mechanics. In: Sanchez-Palencia, E., Zaoui, A. (Eds.), Homogenization Techniques for Composite Media. Vol. 272 of Lecture Notes in Physics. Springer Verlag, New York, pp. 193-278.

Swan, C., 1994. Techniques for stress- and strain-controlled homogenization of inelastic periodic composites. Comput. Methods Appl. Mech. Engrg. 117, 249-267.

Torquato, S., 2003. Random Heterogeneous Materials. Springer-Verlag, Berlin.

Tvergaard, V., 1982. On localization in ductile materials containing spherical voids. Int. J. Fracture 18, $237-252$.

Tvergaard, V., 1990. Material failure by void growth to coalescence. In: Hutchinson, J., Wu, T. (Eds.), Advances in Applied Mechanics. Vol. 27. Academic Press, New-York, pp. 83-151.

Vincent, P.-G., Monerie, Y., Moulinec, H., Suquet, P., 2013. Effective flow surface of porous materials with two populations of voids under internal pressure: I. a GTN model. International Journal of Plasticity , submitted.

Vincent, P.-G., Monerie, Y., Suquet, P., 2009. Porous materials with two populations of voids under internal pressure: I. Instantaneous constitutive relations. International Journal of Solids and Structures 46, 480506.

Zeman, J., Vondřejc, J., Novák, J., Marek, I., 2010. Accelerating a FFT-based solver for numerical homogenization of periodic media by conjugate gradients. Journal of Computational Physics 229 (21), 8065-8071.

\section{Appendix A. Effective flow surface}

The minimization problem (10) is non-smooth in the sense that the function $\varphi$ to be minimized is not differentiable at the origin. Although this minimization problem could be attacked directly (de Buhan and Maghous, 1995; Pastor et al., 2009) with adequate minimization algorithms for nondifferentiable functions, an alternative procedure consists in the resolution of a sequence of evolution problems for a composite with elastic ideally plastic phases (instead of being rigid-plastic). First, each individual phase is given an artificial (or real) elastic stiffness $\boldsymbol{C}(\boldsymbol{x})$ so that the constituents are elastic ideally-plastic. This modification is legitimate since the overall flow surface of the composite does not depend on the elastic properties of the constituents. Second, an artificial time $t$ is introduced. The evolution of the local fields $\boldsymbol{\sigma}(t, \boldsymbol{x}), \boldsymbol{u}^{*}(t, \boldsymbol{x})$ within the unit cell is determined under the application of a strain-rate $\dot{\boldsymbol{E}}(t)$ (which is given in the strain-controlled method or to be determined in the stress-controlled method). For simplicity, the strain-rate is assumed to 
be prescribed and constant $\dot{\boldsymbol{E}}(t)=\dot{\boldsymbol{E}} \forall t \geq 0$.

\section{Appendix A.1. Incremental plasticity}

When an incremental model of plasticity is assumed for the individual constituents, the evolution problem reads as:

$$
\left.\begin{array}{c}
\boldsymbol{\sigma}(t, \boldsymbol{x})=\boldsymbol{C}(\boldsymbol{x}):\left(\boldsymbol{\varepsilon}(\boldsymbol{u}(t, \boldsymbol{x}))-\boldsymbol{\varepsilon}^{p}(t, \boldsymbol{x})\right), \\
\boldsymbol{\sigma}(t, \boldsymbol{x}) \in P(\boldsymbol{x}), \quad \dot{\boldsymbol{\varepsilon}}^{p}(t, \boldsymbol{x})=\dot{\lambda}(t, \boldsymbol{x}) \frac{\partial f}{\partial \boldsymbol{\sigma}}(\boldsymbol{x}, \boldsymbol{\sigma}(t, \boldsymbol{x})), \quad \dot{\lambda} \geq 0, \\
\operatorname{iv}(\boldsymbol{\sigma}(t, \boldsymbol{x}))=0, \quad \boldsymbol{u}(t, \boldsymbol{x})=t \dot{\boldsymbol{E}} \cdot \boldsymbol{x}+\boldsymbol{u}^{*}(t, \boldsymbol{x}), \quad \boldsymbol{u}^{*} \#, \quad \boldsymbol{\sigma} \cdot \boldsymbol{n}-\#,
\end{array}\right\}
$$

where $f(\boldsymbol{x},$.$) is the equation defining the boundary of P(\boldsymbol{x})=\{\boldsymbol{\tau}, f(\boldsymbol{x}, \boldsymbol{\tau}) \leq 0\}$, when such an equation exists, otherwise the normality rule is used in generalized form.

Let $\hat{\boldsymbol{\Sigma}}$ be an element in $P^{\text {hom }}$ and let $\hat{\boldsymbol{\sigma}}(\boldsymbol{x})$ denote the associated local stress field through (9). Taking the time derivative of the first line in (A.1), multiplying it by $\hat{\boldsymbol{\sigma}}-\boldsymbol{\sigma}(t)$ and integrating over the volume element, yields after due use of Hill's lemma:

$$
\left\langle\dot{\boldsymbol{\sigma}}(t): \boldsymbol{C}^{-1}:(\hat{\boldsymbol{\sigma}}-\boldsymbol{\sigma}(t))\right\rangle \geq \dot{\boldsymbol{E}}:(\hat{\boldsymbol{\Sigma}}-\boldsymbol{\Sigma}(t)),
$$

where $\boldsymbol{\Sigma}$ and $\hat{\boldsymbol{\Sigma}}$ are the volume averages of the fields $\boldsymbol{\sigma}$ and $\hat{\boldsymbol{\sigma}}$. Assume that in the limit as $t \rightarrow+\infty, \boldsymbol{\sigma}(t)$ has a limit $\boldsymbol{\sigma}^{\infty}$. Then $\dot{\boldsymbol{\sigma}}$ vanishes and passing to the limit in (A.1) one gets that:

$$
\dot{\boldsymbol{E}}:\left(\hat{\boldsymbol{\Sigma}}-\boldsymbol{\Sigma}^{\infty}\right) \leq 0, \quad \boldsymbol{\Sigma}^{\infty}=\lim _{t \rightarrow+\infty} \boldsymbol{\Sigma}(t) .
$$

Therefore $\boldsymbol{\Sigma}^{\infty}$ is on the boundary of $P^{\text {hom }}$ (it was already known that it was in $P^{\text {hom }}$ as the average of $\boldsymbol{\sigma}^{\infty}$ which satisfies all the requirements of (9)), with a non vanishing outer normal $\dot{\boldsymbol{E}}$ to the boundary of $P^{\text {hom }}$.

\section{Appendix A.2. Deformation theory}

When a deformation theory of plasticity is assumed for the individual constituents, the evolution problem reads exactly as in (A.1), except that the normality rule is written in total form (and not in incremental form) as:

$$
\boldsymbol{\varepsilon}^{p}(t, \boldsymbol{x})=\lambda(t, \boldsymbol{x}) \frac{\partial f}{\partial \boldsymbol{\sigma}}(\boldsymbol{x}, \boldsymbol{\sigma}(t, \boldsymbol{x})), \quad \lambda \geq 0 .
$$

Multiplying the first line of (A.1) by $\hat{\boldsymbol{\sigma}}-\boldsymbol{\sigma}(t)$ and integrating over the volume element, yields after due use of Hill's lemma:

$$
\frac{1}{t}\left\langle\boldsymbol{\sigma}(t): \boldsymbol{C}^{-1}:(\hat{\boldsymbol{\sigma}}-\boldsymbol{\sigma}(t))\right\rangle \geq \dot{\boldsymbol{E}}:(\hat{\boldsymbol{\Sigma}}-\boldsymbol{\Sigma}(t)),
$$

Taking the limit as $t$ tends to $+\infty$ and assuming that $\boldsymbol{\sigma}(t)$ has a limit, yields again:

$$
\dot{\boldsymbol{E}}:\left(\hat{\boldsymbol{\Sigma}}-\boldsymbol{\Sigma}^{\infty}\right) \leq 0, \quad \boldsymbol{\Sigma}^{\infty}=\lim _{t \rightarrow+\infty} \boldsymbol{\Sigma}(t) .
$$


Therefore the asymptotic overall stress $\Sigma^{\infty}$ obtained by the deformation theory belongs to the boundary of $P^{\text {hom }}$ with outer normal $\dot{\boldsymbol{E}}$. Both models, incremental and deformation theory, lead asymptotically to the same overall stress states. However, as is well-known, the path followed to reach these points may be different.

\section{Appendix B. Single void in a von Mises matrix. Simple cubic structure}

To calibrate the spatial resolution used in the numerical simulations, a simple cubic structure is considered first. The volume element is a cube containing a spherical void located at its center. The same void volume fraction as in Richelsen and Tvergaard (1994), $f=0.04$. The matrix is a von Mises material. The influence of the spatial resolution of the image is studied by considering a discretization of the cube into $32^{3}, 64^{3}$ or $128^{3}$ pixels. The results are shown in Figure B.21 for axisymmetric loading $(\omega=0)$ and pure shear $(\omega=\pi / 6)$ where the Lode angle $\omega$ is defined in (3.3). The results are consistent with those obtained by Richelsen and Tvergaard (1994) (these authors also considered other loading conditions corresponding to other values of $\omega$ which are not reported here). The influence of the

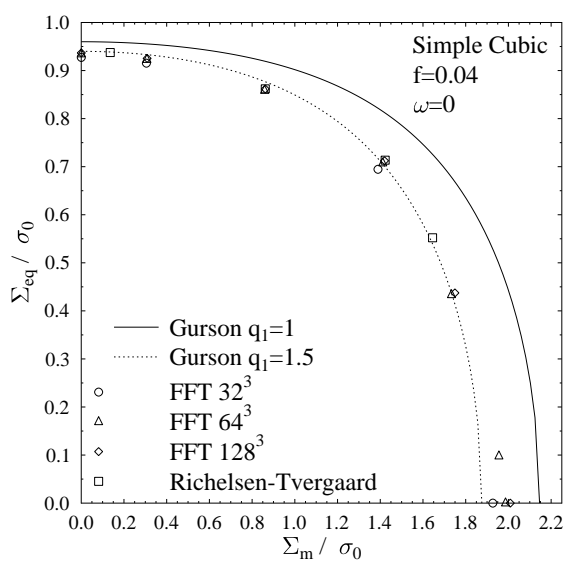

(a)

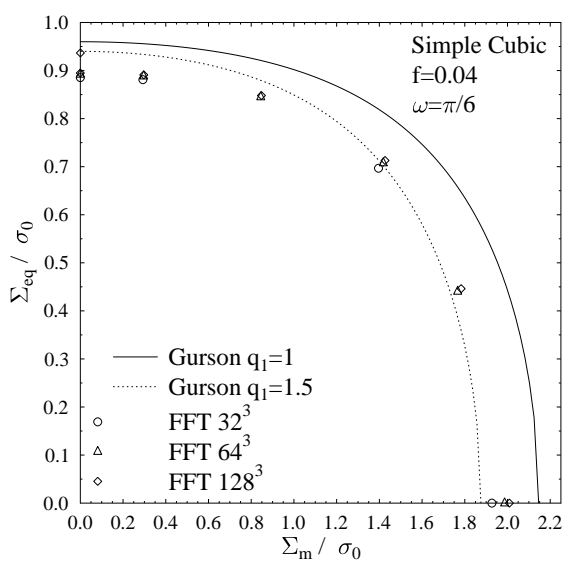

(b)

Figure B.21: Single void in a cubic unit-cell. $f=0.04$. Influence of the spatial resolution. Left: axisymmetric loading $\omega=0$. Right: pure shear $\omega=\pi / 6$.

spatial resolution is relatively small with a maximum when $T=1$ and $T=\infty$. A spatial resolution $64^{3}$ seems to achieve a reasonable compromise between accuracy and the size of the problem.

\section{Appendix C. Deviation from isotropy}




\begin{tabular}{|c|c|c|c|c|c|c|c|c|}
\hline $\mathrm{T}$ & $\# 1$ & $\# 2$ & $\# 3$ & $\# 4$ & $\#$ 5 & \# 6 & Average & Reduced \\
\hline$-\infty$ & 6.49 & 9.52 & 7.65 & 5.16 & 8.17 & 4.78 & 3.28 & 2.50 \\
-20. & 3.18 & $\mathbf{9 . 8 3}$ & 6.66 & $\mathbf{5 . 3 9}$ & 8.10 & 4.26 & 3.26 & $\mathbf{6 . 4 3}$ \\
-4. & 3.11 & 6.22 & 5.90 & 0.76 & 6.53 & 4.04 & 1.26 & 1.44 \\
-2. & 1.69 & 2.05 & 2.20 & 0.70 & 1.42 & 1.18 & 0.23 & 1.43 \\
-1. & 0.62 & 0.74 & 0.39 & 0.62 & 0.43 & 0.29 & 0.07 & 0.46 \\
$-1 / 3$ & 0.31 & 0.37 & 0.11 & 0.50 & 0.15 & 0.18 & 0.03 & 0.15 \\
0. & 0.34 & 0.40 & 0.10 & 0.55 & 0.16 & 0.19 & 0.03 & 0.08 \\
$1 / 3$ & 0.55 & 0.64 & 0.16 & 0.72 & 0.33 & 0.30 & 0.06 & 0.09 \\
1. & 1.67 & 1.94 & 0.64 & 1.25 & 1.08 & 0.85 & 0.18 & 0.42 \\
2. & 3.45 & 5.79 & 2.81 & 0.79 & 2.58 & 1.88 & 0.70 & 1.86 \\
4. & 6.03 & 8.53 & 3.46 & 4.63 & 5.89 & 3.94 & 1.44 & 2.77 \\
20. & $\mathbf{7 . 2 8}$ & 4.70 & 3.77 & 2.52 & 4.40 & 2.09 & 2.29 & 3.26 \\
$+\infty$ & 6.49 & 9.52 & $\mathbf{7 . 6 5}$ & 5.16 & $\mathbf{8 . 1 7}$ & $\mathbf{4 . 7 8}$ & $\mathbf{3 . 2 8}$ & 2.50 \\
\hline
\end{tabular}

Table C.1: Deviation from isotropy in \% measured by $\eta(7)$ for the 6 microstructures of Figure 6 and the microstructure of Figure 2 (right) (Reduced microstructure). Von Mises matrix, $\omega=0$.

\begin{tabular}{|c|c|c|c|c|c|c|c|}
\hline $\mathrm{T}$ & $\# 1$ & $\# 2$ & $\# 3$ & $\# 4$ & $\#$ 5 & $\# 6$ & Average \\
\hline$-\infty$ & 6.49 & 9.52 & 7.65 & 5.16 & 8.17 & 4.78 & 3.28 \\
-20. & 1.98 & 6.21 & 3.09 & 3.71 & 2.49 & $\mathbf{5 . 6 4}$ & 2.88 \\
-4. & 3.29 & 2.56 & 1.89 & 1.38 & 2.03 & 1.24 & 0.57 \\
-2. & 5.53 & 1.60 & 0.63 & 0.72 & 3.37 & 0.52 & 1.38 \\
-1. & 4.40 & 5.81 & 1.83 & 0.97 & 1.81 & 1.65 & 1.60 \\
$-1 / 3$ & 1.82 & 2.18 & 1.62 & 0.79 & 0.65 & 1.50 & 0.63 \\
0. & 0.09 & 0.20 & 0.07 & 0.50 & 0.25 & 0.33 & 0.05 \\
$1 / 3$ & 1.69 & 2.17 & 1.58 & 0.55 & 0.58 & 1.32 & 0.54 \\
1. & 3.76 & 6.40 & 2.12 & 0.98 & 1.32 & 1.76 & 1.26 \\
2. & 3.22 & 8.49 & 3.04 & 0.90 & 1.01 & 2.93 & 1.20 \\
4. & 3.91 & 6.90 & 2.37 & 1.25 & $\mathbf{1 1 . 2 3}$ & 4.04 & 2.88 \\
20. & $\mathbf{7 . 0 8}$ & 4.55 & 4.55 & 3.45 & 4.42 & 1.98 & 1.95 \\
$+\infty$ & 6.49 & $\mathbf{9 . 5 2}$ & $\mathbf{7 . 6 5}$ & $\mathbf{5 . 1 6}$ & 8.17 & 4.78 & $\mathbf{3 . 2 8}$ \\
\hline
\end{tabular}

Table C.2: Deviation from isotropy in $\%$ measured by $\eta(7)$ for the 6 microstructures of Figure 6 and the microstructure of Figure 2 (right) (Reduced microstructure). Von Mises matrix, $\omega=\pi / 6$. 


\begin{tabular}{|c|c|c|c|c|c|c|c|c|}
\hline Microstructure & \multicolumn{2}{|c|}{$\# 1$} & \multicolumn{2}{|c|}{$\# 2$} & \multicolumn{2}{c|}{$\# 3$} & \multicolumn{2}{c|}{ Average } \\
\hline$\omega$ & 0 & $\pi / 6$ & 0 & $\pi / 6$ & 0 & $\pi / 6$ & 0 & $\pi / 6$ \\
\hline$-\infty$ & 6.23 & 6.23 & 9.76 & 9.76 & 9.63 & 9.63 & 2.88 & 2.88 \\
-20. & 5.57 & 3.28 & $\mathbf{1 0 . 4 8}$ & 7.09 & $\mathbf{1 0 . 3 5}$ & 2.52 & 2.69 & 1.96 \\
-4. & 3.92 & 1.52 & 8.86 & 3.08 & 7.14 & 0.8 & 1.64 & 0.91 \\
-2. & 2.38 & 0.83 & 4.5 & 2.35 & 4.18 & 1.04 & 0.78 & 1.11 \\
-1. & 1.23 & 0.99 & 1.99 & 1.77 & 1.87 & 0.81 & 0.43 & 1.01 \\
$-1 / 3$ & 0.44 & 0.61 & 0.92 & 0.47 & 0.53 & 0.72 & 0.25 & 0.52 \\
0. & 0.18 & 0.53 & 0.66 & 0.36 & 0.22 & 0.49 & 0.18 & 0.27 \\
$1 / 3$ & 0.14 & 0.64 & 0.76 & 0.94 & 0.36 & 0.51 & 0.20 & 0.31 \\
1. & 0.39 & 0.78 & 1.97 & 2.21 & 0.84 & 0.63 & 0.66 & 0.79 \\
2. & 0.83 & 1.44 & 3.49 & 3.57 & 1.53 & 1.53 & 1.14 & 1.40 \\
4. & 1.58 & 2.82 & 4.4 & 4.66 & 3.65 & 3.19 & 0.87 & 1.38 \\
20. & 5.49 & 5.39 & 7.72 & 8.21 & 6.38 & 3.4 & 2.80 & $\mathbf{3 . 3 2}$ \\
$+\infty$ & $\mathbf{6 . 2 3}$ & $\mathbf{6 . 2 3}$ & 9.76 & $\mathbf{9 . 7 6}$ & 9.63 & $\mathbf{9 . 6 3}$ & $\mathbf{2 . 8 8}$ & 2.88 \\
\hline
\end{tabular}

Table C.3: Deviation from isotropy in $\%$ measured by $\eta(7)$ for the 3 microstructures of Figure 3 . Von Mises matrix. $\omega=0, \pi / 6$.

\begin{tabular}{|c|c|c|c|}
\hline & \multicolumn{3}{|c|}{$\begin{array}{c}\text { Spherical voids } \\
\text { Reduced microstructure }\end{array}$} \\
\hline $\mathrm{T}$ & $p_{b}=0$ & $p_{b}=0.5 \sigma_{0}$ & $p_{b}=1.5 \sigma_{0}$ \\
\hline$-\infty$ & 2.55 & 2.64 & 2.50 \\
-20. & $\mathbf{5 . 9 5}$ & $\mathbf{6 . 5 0}$ & $\mathbf{6 . 7 5}$ \\
-4. & 1.97 & 3.27 & 1.46 \\
-2. & 1.25 & 2.36 & 1.42 \\
-1. & 0.46 & 0.83 & 0.45 \\
$-1 / 3$ & 0.15 & 0.15 & 0.10 \\
0. & 0.08 & 0.08 & 0.11 \\
$1 / 3$ & 0.10 & 0.13 & 0.39 \\
1. & 0.55 & 0.64 & 1.98 \\
2. & 2.02 & 2.39 & 0.31 \\
4. & 1.90 & 0.50 & 0.94 \\
20. & 2.48 & 2.71 & 0.17 \\
$+\infty$ & 2.55 & 1.74 & 0.27 \\
\hline
\end{tabular}

\begin{tabular}{|c|c|c|c|}
\hline & \multicolumn{3}{|c|}{ Ellipsoidal voids } \\
& \multicolumn{3}{|c|}{ Microstructure \# 0 } \\
\hline $\mathrm{T}$ & $p_{b}=0$ & $p_{b}=0.5 \sigma_{0}$ & $p_{b}=1.5 \sigma_{0}$ \\
\hline$-\infty$ & 5.72 & 2.81 & $\mathbf{6 . 2 0}$ \\
-20. & 5.83 & $\mathbf{5 . 8 0}$ & 5.60 \\
-4. & 3.36 & 4.19 & 3.98 \\
-2. & 2.87 & 0.31 & 2.37 \\
-1. & 1.30 & 1.24 & 1.20 \\
$-1 / 3$ & 0.45 & 0.45 & 0.45 \\
0. & 0.18 & 0.18 & \\
$1 / 3$ & 0.13 & 0.11 & 0.10 \\
1. & 0.42 & 0.47 & 0.85 \\
2. & 0.86 & 0.88 & 1.03 \\
4. & 1.9 & 2.16 & 1.71 \\
20. & 4.87 & 4.17 & 2.41 \\
$+\infty$ & $\mathbf{6 . 0 2}$ & 3.98 & 2.56 \\
\hline
\end{tabular}

Table C.4: Influence of the internal pressure in the voids on the deviation from isotropy in $\%$ measured by $\eta$ (7). Gurson matrix, $\omega=0$. Left: reduced microstructure of Figure 2. Right: microstructure \# 1 of Figure 3 . 


\section{Appendix D. Covariograms}

In order to qualitatively evaluate the stationarity and the isotropy of the image \# 1 (Figure 3), a specific study based on covariograms is presented here. It is recalled that the the image \# 1 contains $512^{3}$ voxels and 511 ellipsoidal voids (monodisperse voids, aspect ratio $1 / 5$, porosity 0.04 ). First of all, an additional image has been built with $1024^{3}$ voxels and 4088 ellipsoidal voids (while keeping the other parameters identical to those of the image \# 1). Given its large size and number of cavities therein, this second image is our "reference image" for stationarity and isotropy that we could get from this type of microstructures.

\section{Appendix D.1. Characterization of the reference image}

Covariograms $C(\boldsymbol{r})$ have been plotted for several cutting planes and in several directions. Results are reported in Figure D.22.

It appears that the covariograms stabilize from about 100 pixels (which is about 3 times the radius of the safety spheres surrounding the voids), with an integral range of about 25 pixels. These values are much lower than the total size of the unit-cell, suggesting that the stationarity has been reached for each of these cutting planes.

Moreover, in each of these planes, the obtained covariograms remain close to each other, meaning that they are independent of the orientation: $C(\boldsymbol{r}) \approx C(\|\boldsymbol{r}\|)$.

These two last observations will serve as benchmarks with respect to the stationarity and global isotropy that could be obtained from such images.

\section{Appendix D.2. Characterization of the image \# 1 (Figure 3)}

Covariograms have been plotted and results are reported in Figure D.23.

As for the reference image, it appears that: (1) the covariograms stabilize from about 100 pixels, with an integral range of about 25 pixels, (2) for each cut, the covariograms remain close to each other $(C(\boldsymbol{r}) \approx C(\|\boldsymbol{r}\|))$. Consequently, the same features as in the reference image are found, suggesting that the two images have approximately the same degree of stationarity and isotropy.

Moreover, as expected in the case of stationary random close sets, the variance of the surface fraction of the inclusions (here the voids) is decreasing with the size of the cutting planes (Stoyan et al., 1995): Figure D.24 shows this variance for 100 parallel cutting planes as a function of the size of the samples inside these planes. One can also observe that the variance is very low (less than $10^{-4}$ ) from a size of 300 pixels, suggesting a good level of isotropy for the image \# 1 with 512 pixels length (it would be 0 if this image were perfectly isotropic). Finally, for the present study, simulations have been performed on $512^{3}$ voxels images and not on $1024^{3}$ voxels images, ensuring reasonable computation costs (about 35 hours on 20 cores and 70 GB RAM).

This covariogram based analysis remains mostly qualitative. Concerning the problem of global isotropy, the mechanical approach introduced in the section 2.4 leads to a quantitative measure of the deviation from isotropy. 

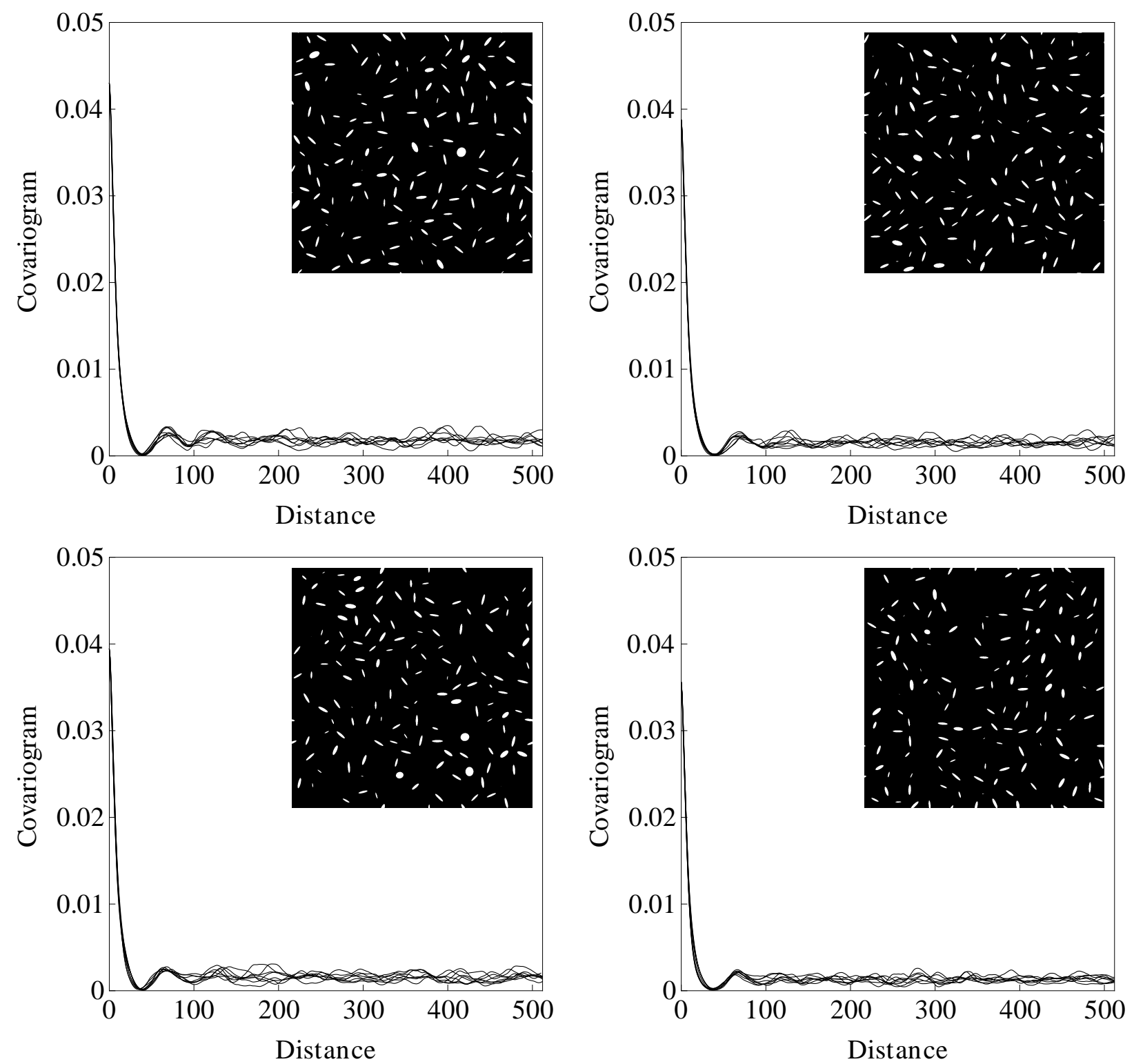

Figure D.22: Covariograms in terms of distance (in pixels). 4 parallel cutting planes in the $1024^{3}$ voxels image (with 4088 voids). On each of these cutting planes, covariograms are plotted in 8 directions. Cutting planes are also reported on each figure. 

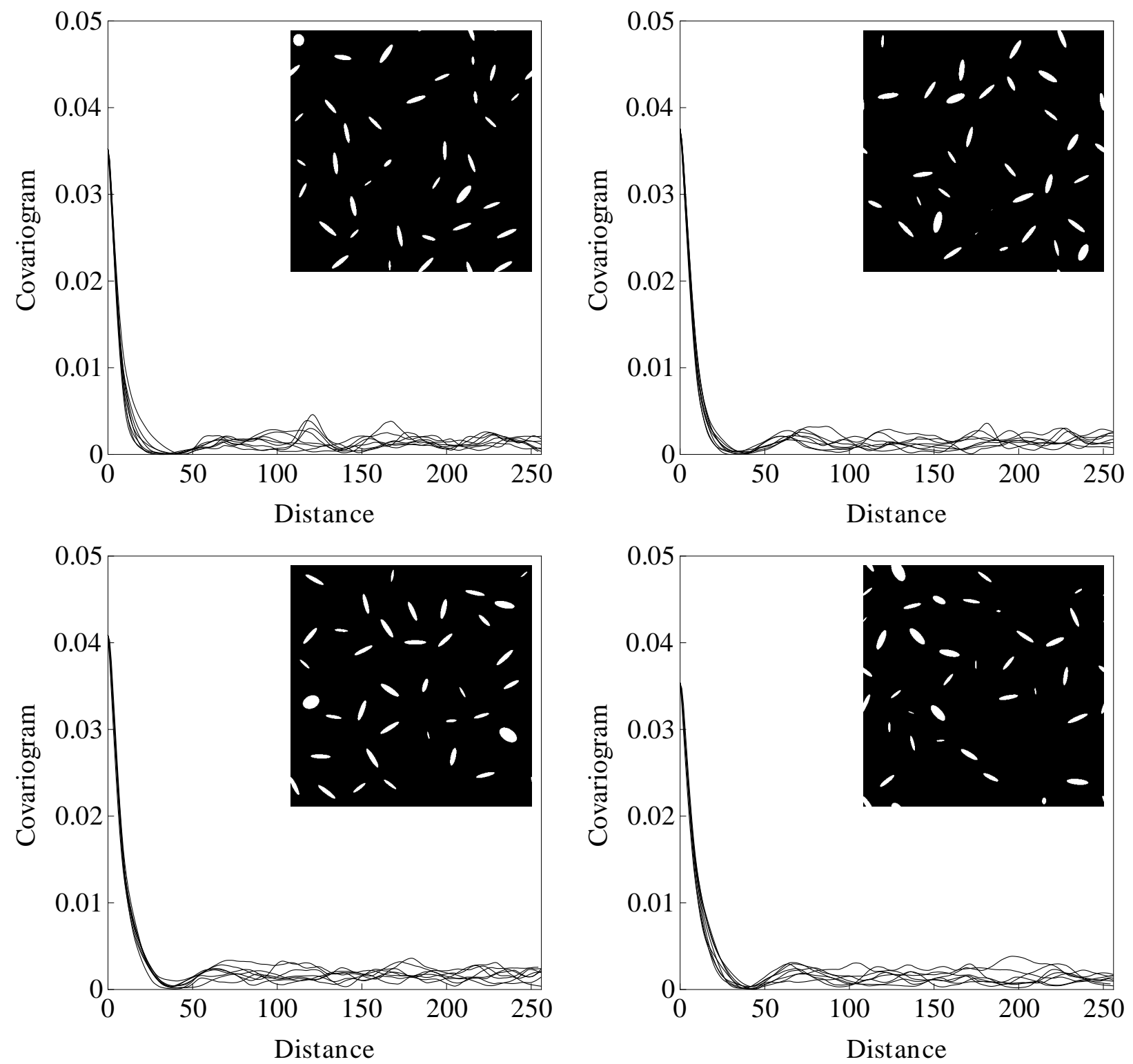

Figure D.23: Covariograms in terms of distance (in pixels). 4 parallel cutting planes in the image \#1 (Figure 3). On each of these cutting planes, covariograms are plotted in 8 directions. Cutting planes are also reported in each figure. 


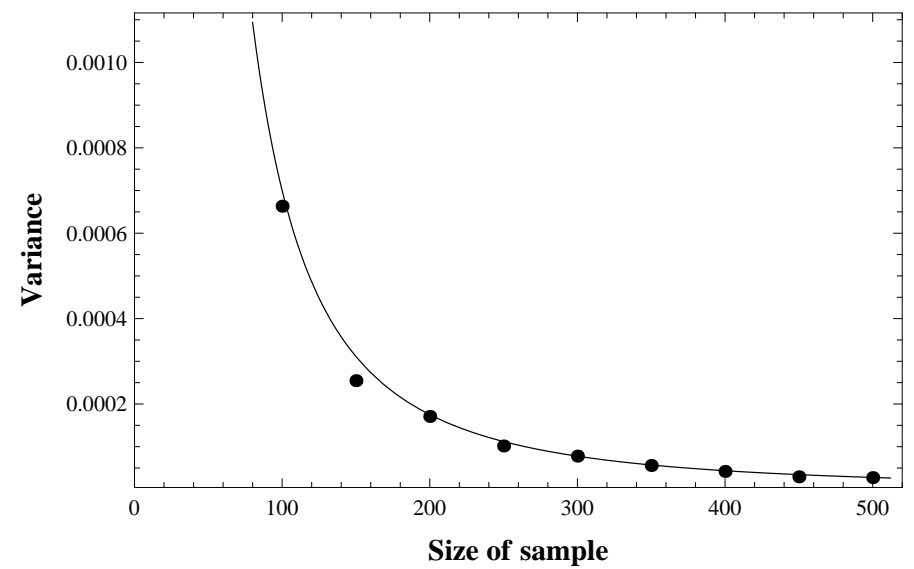

Figure D.24: Image \#1 (Figure 3): variance of the surface fractions for 100 parallel cutting planes as a function of the size of the samples inside these planes (in pixels). 